\title{
Design of Optimal Proportional Integral Derivative Based Power System Stabilizer Using Bat Algorithm
}

\author{
Dhanesh K. Sambariya ${ }^{1}$ and Rajendra Prasad ${ }^{2}$ \\ ${ }^{1}$ Department of Electrical Engineering, Rajasthan Technical University, Kota, Rajasthan 324010, India \\ ${ }^{2}$ Department of Electrical Engineering, Indian Institute of Technology Roorkee, Roorkee, Uttaranchal 247667, India \\ Correspondence should be addressed to Dhanesh K. Sambariya; dsambariya_2003@yahoo.com
}

Received 7 November 2015; Accepted 1 February 2016

Academic Editor: Meng J. Er

Copyright ( 2016 D. K. Sambariya and R. Prasad. This is an open access article distributed under the Creative Commons Attribution License, which permits unrestricted use, distribution, and reproduction in any medium, provided the original work is properly cited.

\begin{abstract}
The design of a proportional, derivative, and integral (PID) based power system stabilizer (PSS) is carried out using the bat algorithm (BA). The design of proposed PID controller is considered with an objective function based on square error minimization to enhance the small signal stability of nonlinear power system for a wide range of operating conditions. Three benchmark power system models as single-machine infinite-bus (SMIB) power system, two-area four-machine ten-bus power system, and IEEE New England ten-machine thirty-nine-bus power system are considered to examine the effectiveness of the designed controller. The BA optimized PID based PSS (BA-PID-PSS) controller is applied to these benchmark systems, and the performance is compared with controllers reported in literature. The robustness is tested by considering eight plant conditions of each system, representing the wide range of operating conditions. It includes unlike loading conditions and system configurations to establish the superior performance with BA-PID-PSS over-the-counter controllers.
\end{abstract}

\section{Introduction}

Power system stability is an ability to regain synchronism on occurrence of disturbance. In general, an electric power system (EPS) is large, complex in nature, and interconnected and prone to small signal oscillation on occurrence of disturbances. These low-frequency electromechanical oscillations (EMOs) persist because of insufficient damping torque caused by high/adverse operating conditions. In the absence of sufficient damping, the EMOs may persist for longer time resulting in limitations on power transfer capability of EPSs. In multimachine EPS model, two distinct types of EMOs are recognized [1]. The oscillations associated with generators at a generating station, swinging with respect to the rest of the power system, are called intra-area mode oscillations.

Similarly, the swinging of many machines in an area of EPS against machines in another area is called interarea oscillations. Thus, a supplementary control signal is added to the excitation system to damp out these oscillations, and the system is called the power system stabilizer (PSS) [2]. The widely used PSS is conventional PSS, which is designed by phase compensation in the frequency domain and is introduced as lead lag compensator. It is necessary to have a linearized model of EPS to design CPSS parameters by using modern control techniques, such as to provide well damping for both types of oscillations. The CPSS parameters are tuned to an operating point which may fail to give satisfactory damping to other operating conditions within the power system. The use of adaptive control techniques to design CPSS may eliminate this limitation but is complex in nature and costly $[3,4]$.

In recent years, many optimization methods based on random search are suitable for solving complex problems, which are impossible to be solved by mathematical methods such as the gradient. Application of new optimization methods and fuzzy and intelligent method is the focus of researchers to design a good quality controller for enhancement of small signal stability of a power system $[5,6]$.

In [7], the fuzzy logic based power system stabilizer is designed for single-machine infinite-bus power system model and extended it to multimachine power system. To mitigate the shortcomings of conventional methods many 
optimization based algorithms have been proposed. The methods available in literature are Tabu search [8], evolutionary algorithm [9], the differential evolution (DE) algorithm [2], simulated annealing [10], genetic algorithm [11], fuzzy logic with genetic algorithm [12], fuzzy logic with harmony search algorithm [13], interval type-2 fuzzy logic controller, different membership function based fuzzy controller, artificial bee colony (ABC) [14], particle swarm optimization $[15,16]$, robust fast output sampling feedback [17], and an iterative linear matrix inequalities algorithm [18].

The different controller structures are always the field of interest for researchers. The proportional integral derivative (PID) type PSS is used for improving damping of EPSs. This is generally accepted in the industries for various applications. In literature several PID based PSSs are designed for SMIB power system such as using genetic algorithm (GA) [19, 20], harmony search algorithm (HSA) $[19,21]$, bacterial foraging algorithm (BFA) [22, 23], real coded genetic algorithm (RCGA) [24], Ziegler-Nichols (ZN) [25], hybrid particle swarm-bacteria foraging optimization (PSO-BFA) [25], artificial bee colony (ABC) [26], and biogeographical based optimization (BBO) [27] and for two-area four-machine tenbus power system such as iterative linear matrix inequality (ILMI) [18] based PID based PSS which is successfully employed. The application of GA has been reported to tune the PI and PID based PSS design for multimachine power systems [20, 28].

To mitigate the drawbacks of above optimization methods for PSS design, a relatively new optimization scheme known as the bat algorithm (BA) is used for the PID type PSS parameter design. It appeared as a promising one for handling the optimization problems even with epistatic objective functions as in ten-machine power systems where the number of variables is ranging up to 27. It is not largely affected by the size and nonlinearity of the problem and can converge to the optimal solution in many cases where many analytical methods fail to converge. Considering the strength of this algorithm, it is employed in the present work for the optimal PID tuning for stability enhancement in SMIB, fourmachine, and ten-machine power systems.

In the organization of the paper, the problem is formulated in Section 2 with information about power system models, on PID and about the objective function used in optimization. Section 3 includes the detail on bat algorithm. The PID parameters tuning scheme and results are included for three power systems in Section 4. The simulation results and eigenvalue analysis is carried out with proposed BA-PIDPSS for SMIB, four-machine, and ten-machine power systems in Sections 4.1, 4.2, and 4.3, respectively. Lastly, the analysis is concluded in Section 5.

\section{Problem Formulation}

The aim of the paper is to utilize the bat algorithm for tuning the PID parameters in the power system; therefore, the EPS elements such as generators, excitation system, and PSS must be modeled. To complete the tuning process, an objective function to obtain satisfactory results is necessary and should

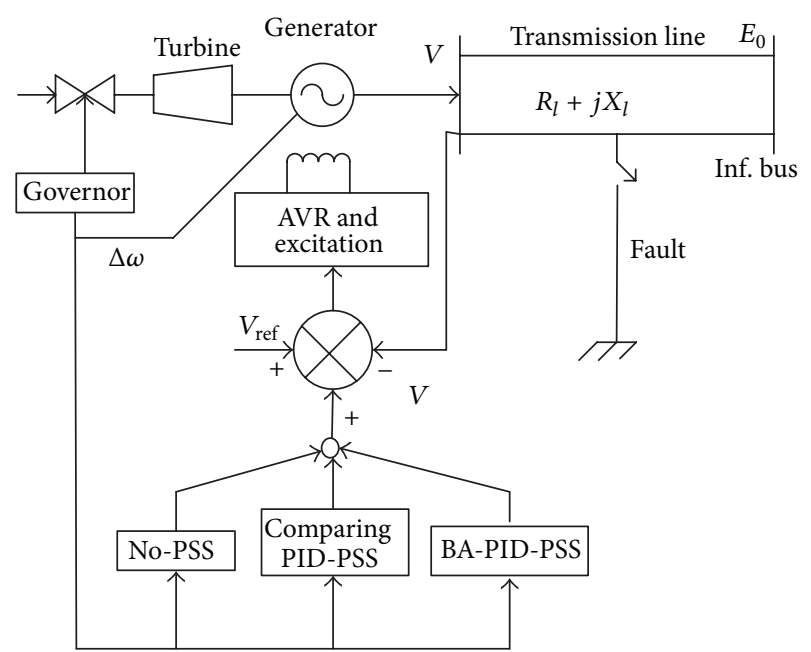

FIGURE 1: Line diagram of single-machine infinite-bus power system.

be defined. Therefore, the system model and an objective function used in PSS parameter tuning in a multimachine power system are elaborated.

2.1. Test System Configuration. The systems under consideration are single-machine connected to infinite-bus (SMIB) power system, two-area four-machine ten-bus power system, and IEEE New England 10-machine 39-bus power system. The general representation of a power system using nonlinear differential equations can be given as in the following equation:

$$
\dot{X}=f(X, U),
$$

where $X$ and $U$ represent the vector of state variables and the vector of input variables, respectively. As in [29], the power system stabilizers can be designed by use of the linearized incremental models of the power system around an operating point. The general representation of a power system can be written in terms of state equations as in

$$
\Delta \dot{X}=\Delta X+B U
$$

Moreover, a brief introduction of the considered systems is given in the next sections.

2.1.1. SMIB Power System. The single line diagram representation of single-machine connected to infinite-bus power system is shown in Figure 1. The connection of transmission line to generator, automatic voltage regulator, excitation system, and power system stabilizer is shown in this figure [29-31]. The linearized model called Heffron-Phillip model is presented in Figure 2. The scheme of sensing signals and the optimization scheme are also shown in this figure. The considered synchronous machines of SMIB and multimachine power systems are of model 1.0 type as discussed in [2]. To cover all operating conditions, the power system with generators, stabilizers, and excitation systems can be modeled by a set of nonlinear differential equations as in (1) [32]. 


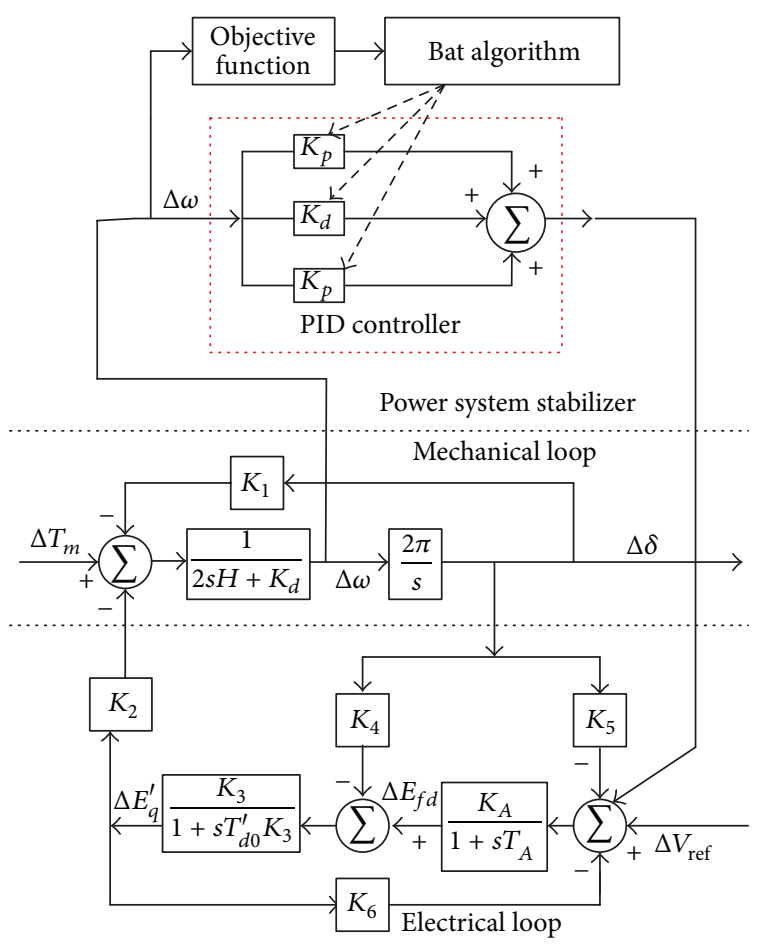

Figure 2: Representation of Heffron-Philip model with PID type PSS and tuning scheme using bat algorithm.

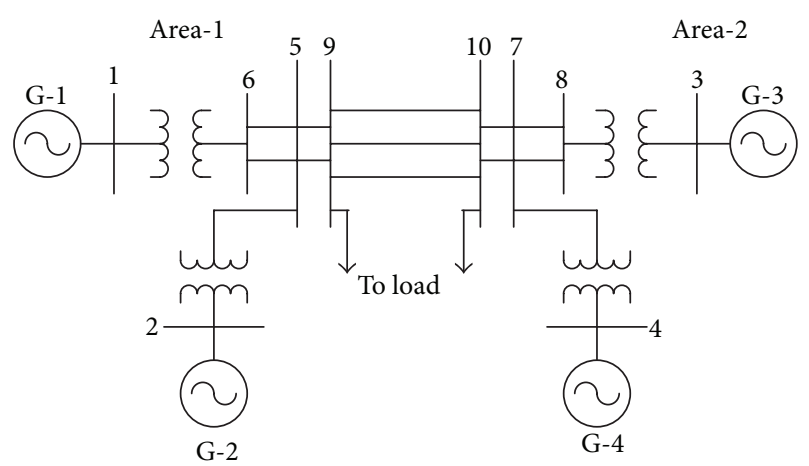

Figure 3: Line diagram representation of four-machine ten-bus power system.

It is necessary to have linearized model of power system around an operating point to analyze the small signal stability of a power system and consequently to design power system stabilizer. Thus, power system represented by (1) is linearized around an equilibrium operating point of the power system and represented by $(2)[5,32]$. As state space model is represented by (2) and consequently the system matrix $A$, therefore, the total electromechanical modes of the system can be evaluated in the form $\lambda=\sigma \pm j \omega$.

2.1.2. 4-Machine 10-Bus Power System. The single line diagram representation of a four-machine ten-bus power system is shown in Figure 3 as in [13,33]. Moreover, a general Heffron-Philip representation is shown in Figure 4. The considered synchronous machine is of the model 1.0 type as discussed in [34]. The power system with generators, stabilizers, and excitation systems can be modeled by a set of nonlinear differential equations as in (1). It is necessary to have linearized model of a power system around an operating point to analyze the small signal stability of a power system and consequently to design power system stabilizer. The power system represented by (1) is linearized around an equilibrium operating point within the power system and represented by $(2)$ as in $[32,35]$. The necessary data considered to fabricate the system in MATLAB are taken as in $[1,35]$.

The state equations of a power system, consisting of $N$ number of generators and $N_{\text {pss }}$ number of power system stabilizers, can be written as in (2), where $A$ is the system matrix of an order as $4 N \times 4 N$ and is given by $\delta f / \delta X$, while $B$ is the input matrix with order $4 N \times N_{\text {pss }}$ and is given by $\delta f / \delta U$. The order of state vector is $4 N \times 1$ and the order of control vector is $N_{\text {pss }} \times 1$.

2.1.3. IEEE 10-Machine 39-Bus Power System. The power system model is prepared from the data as available in [1]. The number of generators is ten, and the 10th generator is considered as slack. Therefore, the controllers are only connected to the remaining nine generators to enhance the small signal stability with proposed methodology. The line diagram of the system is shown in Figure 5 and is fabricated in MATLAB 2011b. The state equations of a power system consist of $N$ number of generators and $N_{\text {pss }}$ number of power system stabilizers but $N_{\text {pss }}$ is 9 in this case, where $A$ is the system matrix of an order as $4 N \times 4 N$ and is given by $\delta f / \delta X$, while $B$ is the input matrix with order $9 N \times N_{\text {pss }}$ and is given by $\delta f / \delta U$. The order of state vector is $9 N \times 1$ and the order of control vector is $N_{\text {pss }} \times 1$.

2.2. On Proportional Integral Derivative PSS. The proportional integral derivative (PID) controller is most popularly known and used as a feedback controller in the field of complex process industries. It can provide excellent robust control performance over a wide range of operating conditions of a power system because of its three different modes of operation. The proportional controller mode can reduce the rise time but is unable to reduce the steady-state error of the response. The higher value of proportional gain may cause a system to become unstable but lower value makes it insensitive or lesser sensitive to even large value of error. The derivative control mode enhances the system stability by reducing overshoot and improving transient response. The integral control mode of operation may eliminate the steady-state error but may worsen the transient response of a system. The lower value of integral gain value makes a system sluggish while the higher value causes a random increase in the overshoot. Therefore, to design the PID controller, all three gains require special attention to get the control signal by the trial-and-error method based on the experience and plant behaviour. The block diagram representation of PID controller for a closed-loop system is shown in Figure 2 and mathematically represented by (3). The notations $\Delta \omega(t)$ 


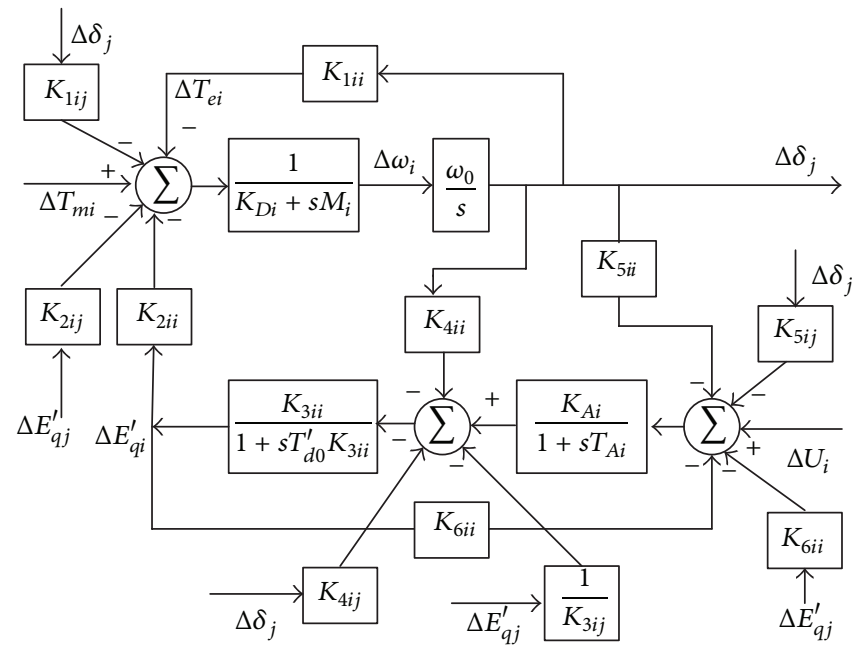

Figure 4: Heffron-Philip model representation for multimachine power systems.

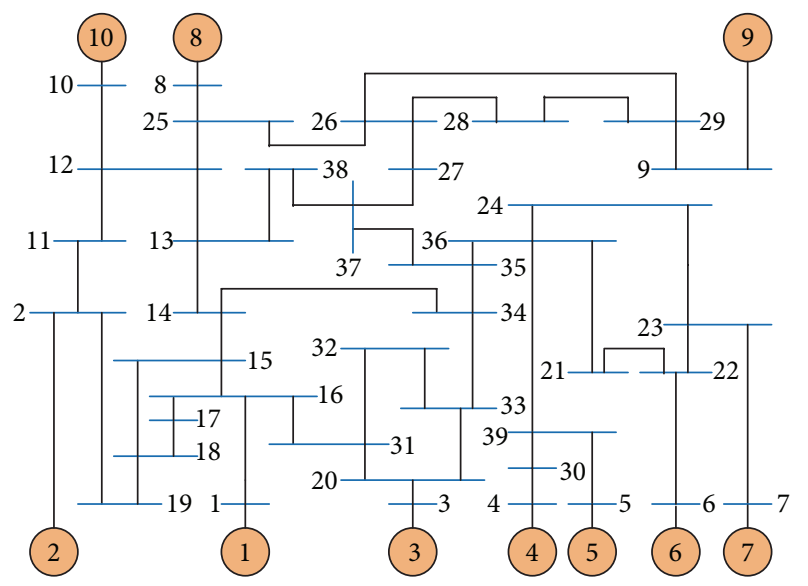

FIGURE 5: Line diagram representation of IEEE New England 10machine 39-bus power system.

represent the change in speed sensed from the rotor shaft of the generator and $T_{\text {sim }}$ refers to simulation time

$$
U_{\mathrm{PID}}=K_{p} \Delta \omega(t)+K_{i} \int_{t=0}^{T_{\text {sim }}} \Delta \omega(t) d t+K_{d} \frac{\partial \Delta \omega(t)}{\partial t}
$$

2.3. Objective Function. To optimize the PID parameters $\left(K_{p}\right.$, $K_{d}$, and $K_{i}$ ) an objective function is formulated, where the damping is maximized in terms of reduced overshoots and settling time in system oscillations. As in integral square error (ISE), the error is heavily reduced/penalized in beginning (during large errors) and low for light errors. Since the speed deviation of the generator is sensed from the shaft of the generator, as an objective function, the ISE based cost function is represented for SMIB, four-machine, and tenmachine power system by (4)-(6), respectively:

$$
\begin{aligned}
& J=\int_{0}^{T_{\text {sim }}}|\Delta \omega(t)|^{2} d t, \\
& J=\sum_{j=1}^{4} \int_{0}^{T_{\text {sim }}}\left|\Delta \omega_{j}(t)\right|^{2} d t, \\
& J=\sum_{j=1}^{10} \int_{0}^{T_{\text {sim }}}\left|\Delta \omega_{j}(t)\right|^{2} d t .
\end{aligned}
$$

Subjected to parameter bounds for SMIB power system as in (7) and for multimachine power systems as in (8). In (8), the operator $j$ refers to the number of PSSs connected in system. It is 4 in 4 -machine system but is 9 in 10-machine power system

$$
\begin{aligned}
& K_{p}^{\min } \leq K_{p} \leq K_{p}^{\max }, \\
& K_{d}^{\min } \leq K_{d} \leq K_{d}^{\max }, \\
& K_{i}^{\min } \leq K_{i} \leq K_{i}^{\max }, \\
& K_{j p}^{\min } \leq K_{j p} \leq K_{j p}^{\max }, \\
& K_{j d}^{\min } \leq K_{j d} \leq K_{j d}^{\max }, \\
& K_{j i}^{\min } \leq K_{j i} \leq K_{j i}^{\max },
\end{aligned}
$$

$$
j=1, \ldots, N_{\mathrm{pss}},
$$

Typical ranges of the optimizing parameters as selected are mentioned in preceding sections for respective power system models. Considering one of the above objectives, the proposed approach employs BA to solve this optimization problem for an optimal set of PSS parameters. The process of optimization of PID parameters is carried out according to the arrangement as in Figure 2, wherein generators of the 
test system are equipped with PID controllers with input as change in speed at the generator shaft. The used bat algorithm is introduced in Section 3.

\section{Review on Bat Algorithm}

This algorithm is based on the echolocation behaviour produced by natural bats in locating their prey. The pulse generated by microbats lasts for 8-10 seconds with frequency range of $25-150 \mathrm{kHz}$ and with associated wavelength of 2$14 \mathrm{~mm}$. Necessary assumptions are required to be considered during development of the echolocation characteristics of microbats [36, 37].

(i) The bats are able to differentiate/detect the prey and background barriers in the search path using echolocation behaviour.

(ii) Assume that $r$ th bat is randomly moving with velocity, location, frequency, wavelength, and intensity represented by $v_{r}, x_{r}, f_{\min }, \lambda_{r}$, and $A^{0}$, respectively. The pulse frequency is regulated and the pulse rate is adjusted in the range $\mathrm{pr}=[0,1]$ on the basis of distance of the prey.

(iii) The loudness of the pulse is adjusted according to the distance of the prey as $A^{0}$ (maximum for large distance) to $A_{\min }$ (minimum for lower distance).

In optimization problems, an objective function is represented by minimization of $F(r)$ and subjected to $x_{r} \in X_{r}$, $r=1,2, \ldots, n$. In initialization step of the bat algorithm, the bat population is generated with velocity $v_{r}$ and position $x_{r}$ for $r=1,2, \ldots, n$. The pulse frequency is selected in the range $f_{r} \in\left[f_{\min }, f_{\max }\right]$. Pulse rate and the loudness are set as above, while the search loop is set to maximum iteration counts as $t \leq T_{\max }$.

In step-2, the new solutions are generated by considering the following equations of frequency, velocity, and position. For $r$ th bat, the new position and velocity at time step $t$ are represented by $x_{r}^{t}$ and $v_{r}^{t}$, respectively:

$$
\begin{aligned}
& f_{r}=f_{\min }+\left(f_{\max }-f_{\min }\right) \beta, \\
& v_{r}^{t}=v_{r}^{t-1}+\left(x_{r}^{t-1}-x^{\prime}\right) f_{r}, \\
& x_{r}^{t}=x_{r}^{t-1}+v_{r}^{t},
\end{aligned}
$$

where $\beta$ represents the uniform distribution in the range $\beta \epsilon$ $[0,1]$. The value of $x^{t}$ represents the best location in the search step for $n$ bats.

In step-3, the local search is applied for the generation of the new solutions using local random walk behaviour as described by the following equation. $\epsilon$ is selected in the range of $[-1,1]$ with average value of loudness $A^{t}$ at time $t$

$$
x_{\text {new }}=x_{\text {old }}+\epsilon A^{t} \text {. }
$$

In step-4, the loop operation for generation of the new solutions is considered. On advancement of iterations, the loudness and the rate of pulse emission have to be updated
TABLE 1: Plant configurations for SMIB power system.

\begin{tabular}{lcc}
\hline PS model & Active power & Reactance \\
\hline Plant-1 & 0.5 & 0.2 \\
Plant-2 & 0.5 & 0.4 \\
Plant-3 & 0.75 & 0.2 \\
Plant-4 & 0.75 & 0.4 \\
Plant-5 & 1.0 & 0.2 \\
Plant-6 & 1.0 & 0.4 \\
Plant-7 & 1.2 & 0.4 \\
Plant-8 & 1.5 & 0.2 \\
\hline
\end{tabular}

by (11). The rate of pulse emission is increased on shortening of the path to prey

$$
\begin{gathered}
A_{r}^{t+1}=x_{\text {old }}+\alpha A_{r}^{t}, \\
\operatorname{pr}_{r}^{t+1}=\operatorname{pr}_{r}^{0}\left[1-e^{-\gamma t}\right],
\end{gathered}
$$

where $\alpha$ and $\gamma$ represent the constant values in the range of $0 \leq \alpha \leq 1$ and $0<\gamma \cdot \alpha$ behaves like the cooling factor of a cooling schedule in the simulated annealing presented in [38]. The generally selected value of these constants is 0.9 as reported by [39].

In last step-5, the stopping criterion is checked as maximum count of iterations is reached and termination of computation is executed. Otherwise, go to steps-3-4 to repeat the process.

\section{Results and Discussion}

\subsection{SMIB Power System}

4.1.1. Simulation Plant Creation. In this section, the performance from the SMIB power system is to be examined with proposed BA-PID-PSS. A block diagram in Simulink, including all the nonlinear blocks, is generated in MATLAB Software. The separate sets of operating conditions of SMIB system are considered based on different combinations of active power $P_{g 0}$ and transmission line reactance $\left(X_{l}\right)$. The intention is to show robust operation of the proposed controller. The eight different sets of operating conditions are termed as plants and details are mentioned in Table 1. The plants are covering light load conditions to heavy load conditions. The nonlinear operation within the system is ensured by applying self-clearing three-phase fault as in $[1$, $4,35]$.

4.1.2. PID Based PSS Design Using Bat Algorithm. In order to assess effectiveness of the proposed bat algorithm, the problem is considered as optimization, to find the PID based PSS parameters. The problem is formulated in MATLAB environment and executed on Intel (R) Core (TM), 2 Duo CPU T6400@2.00 GHz with 3GB RAM, 32-bit operating system. Bat algorithm and its main steps for optimization are mentioned in Section 3. The generally opted initializing values of intensity $(A)$ and pulse rate $(r)$ are considered as 0.5 and 0.5 , respectively, as presented in [40]. By hit and trial 
TABLE 2: Comparison of PID based PSS parameters for SMIB power system.

\begin{tabular}{lccc}
\hline Controller & $K_{p}$ & $K_{d}$ & $K_{i}$ \\
\hline GA-PID-PSS [19] & 9.984 & 8.784 & 1.722 \\
HS-PID-PSS [19] & 26.758 & 15.479 & 3.1202 \\
BFA-PID-PSS [22] & 31.58 & 32.32 & 6.3202 \\
RCGA-PID-PSS [24] & 49.9740 & 7.8802 & 5.6789 \\
ZN-PID-PSS [25] & 30.0 & 2.8 & 3.226 \\
BFA-PID-PSS [23] & 36.1976 & 5.2715 & 1.2732 \\
PSBFA-PID-PSS [23] & 190.4623 & 11.2516 & 2.4753 \\
ABC-PID-PSS [26] & 26.03226 & 0.8354 & 14.7003 \\
BBO-PID-PSS [27] & 51.4655 & 9.6729 & 20.3237 \\
BA-PID-PSO & 23.8668 & 5.0211 & -19.6162 \\
\hline
\end{tabular}

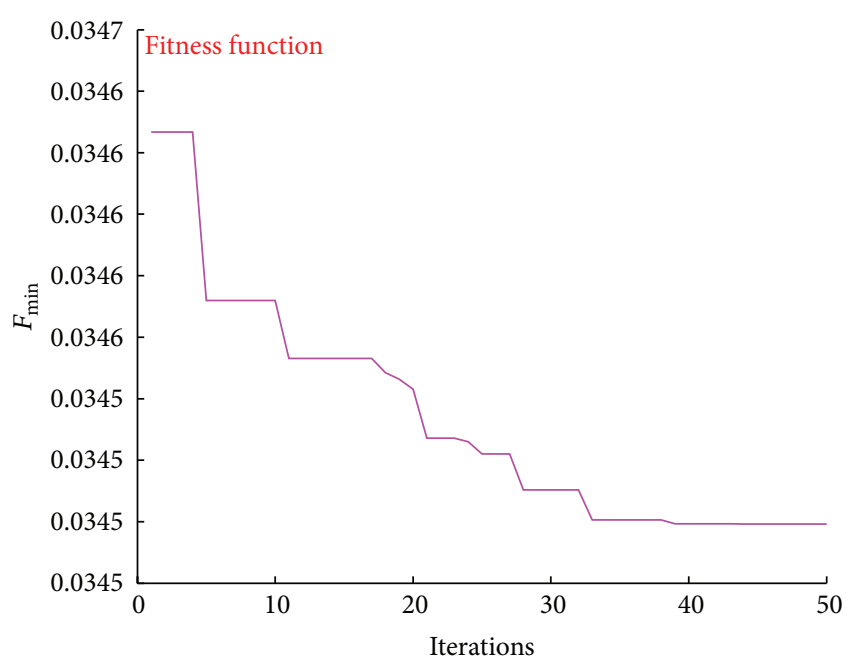

FIGURE 6: Fitness function variation with iteration in tuning of PIDPSS using bat algorithm for SMIB power system.

method, it is found that the suitable values of loudness and pulse rate for PID-PSS optimization using a bat algorithm are as $A=0.9$ and $r=0.1$. The other constraint such as initializing population is selected as $n=25$ and the bandwidth is considered as $f_{\min }=0$ and $f_{\max }=2.0$. The plant (SMIB power system) operating at nominal operating condition (where $X_{l}=0.4 \mathrm{pu}$ and $P_{g 0}=1.0 \mathrm{pu}$ ) is considered for optimal tuning of PID-PSS parameters, subjected to the time domain based simple ISE minimization type objective function with the parametric bounds as $0.001 \leq K_{j p} \leq 70.0$, $0.001 \leq K_{j d} \leq 20.0$, and $-25.0 \leq K_{i j} \leq 25.0$. The plot of fitness function for 50 iterations is shown in Figure 6. The plot for BA-PID-PSS optimized parameters is shown in Figure 7 for the same 50 iterations. It can be seen that the 40 iterations are sufficient because all three parameters are almost constant at 40 and above iterations. The values of PID parameters optimized using bat algorithm are included in Table 2 .

4.1.3. PID Based PSS Reported in Literature. In literature several PID based PSSs are designed for SMIB power system such as using genetic algorithm (GA) [19], harmony search algorithm (HSA) [19], bacterial foraging algorithm (BFA)

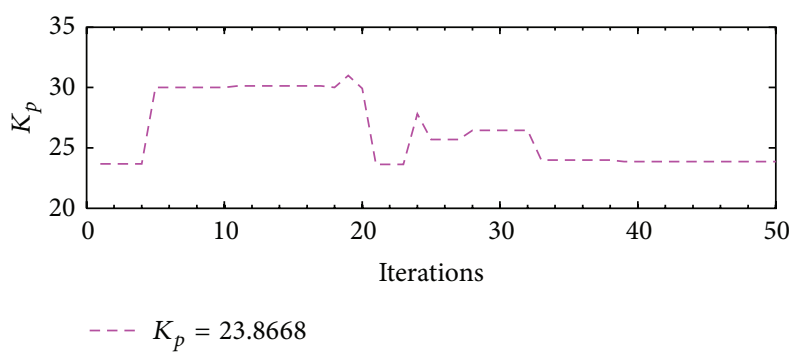

(a)

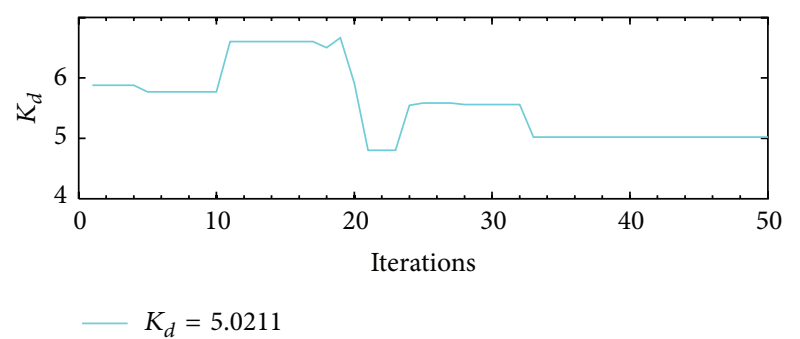

(b)

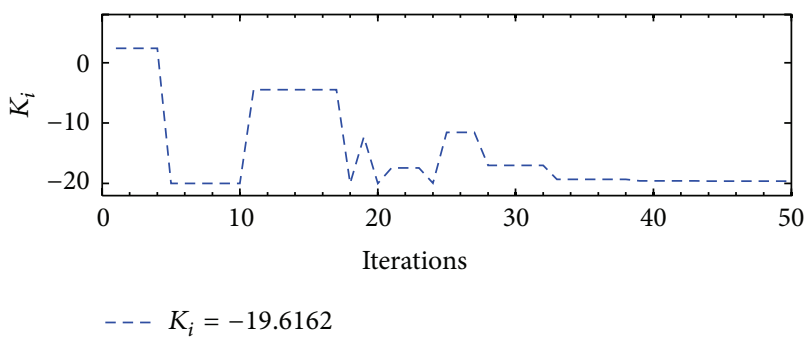

(c)

FIGURE 7: Plot of PID-PSS parameters with iteration count using bat algorithm for SMIB power system.

[22, 23], real coded genetic algorithm (RCGA) [24], ZieglerNichols (ZN) [25], hybrid particle swarm-bacteria foraging optimization (PSO-BFA) [23], artificial bee colony (ABC) [26], and biogeographical based optimization (BBO) [27], and the parameters of the controller are enlisted in Table 2.

4.1.4. Speed Response Analysis. The performance of the SMIB power system is examined under nonlinear mode by creating self-clearing fault at time 5 seconds and persistent for 0.1 seconds. The system with distinct combinations of different active power and transmission line reactance as in Table 1 (eight different plants) and system data is as follows: $x_{d}=$ $1.97 \mathrm{pu}, X_{q}=1.9 \mathrm{pu}, X_{d}^{\prime}=0.30 \mathrm{pu}, T_{d 0}^{\prime}=6.84, K_{A}=$ $100, T_{A}=0.02 \mathrm{sec}, E_{f d}^{\min }=-5 \mathrm{pu}, E_{f d}^{\max }=5 \mathrm{pu}$, and $\omega_{0}=2 \pi \times 50 \mathrm{rad} / \mathrm{sec}$. These eight plants are examined for the speed response with PID based PSSs reported in literature and that with proposed BA-PID-PSS. A Simulink based block diagram, including all the nonlinear blocks, is generated in MATLAB Software. The speed signal is taken as output. Initially, the speed response of the SMIB power system with nominal operating condition (Plant-6) is compared and shown in Figure 8, using BA-PID-PSS (proposed) and the GA-PID-PSS [19], HS-PID-PSS [19], BFA-PID-PSS [22], and 


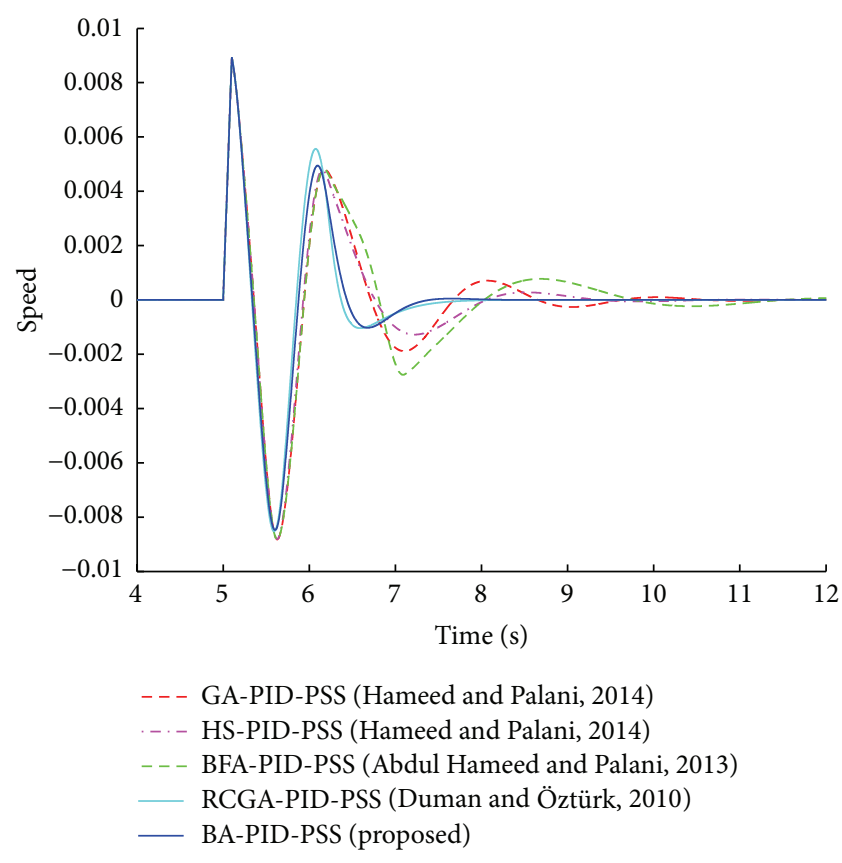

FIGURE 8: Speed response comparison of GA [19], HS [19], BFA [22], RCGA [24], and BA (proposed) based PID type PSS for SMIB power system with nominal operating conditions.

RCGA-PID-PSS [24] as mentioned in Table 2. The speed response of ZN-PID-PSS [25], BFA-PID-PSS [23], PSBFAPID-PSS [23], ABC-PID-PSS [26], BBO-PID-PSS [27], and BA-PID-PSS (proposed) is compared in Figure 9. To observe the robustness of the BA-PID-PSS, the SMIB power system is simulated with the eight plants conditions as in Table 1. The speed response and generator terminal voltage of the SMIB system with BA-PID-PSS are recorded and shown in Figures 10 and 11, respectively. It is easily concluded that the SMIB power system with BA-PID-PSS produces a stable response even with adverse operating conditions (Plant-7 and Plant-8) of the power system. To prove superiority of the BA-PID-PSS, the SMIB system is simulated one by one with other controllers as reported in literature (Table 2) and the performance indices (PIs) of speed response are recorded for the simulation time as 40 seconds and produced in Table 3. The detail on performance indices may be referred from [13]. As the lower value of PI represents the superior performance of the system with reduced settling time and overshoot, in Table 3, the value of PIs (i.e., ITAE, IAE, and ISE are 0.0371, 0.0065 , and $3.4499 \times 10^{-05}$, resp.) with BA-PID-PSS is lesser as compared to others, resulting in good performance, that is, the small signal stability enhancement as compared to others.

4.1.5. Eigenvalue Analysis. In previous section, the speed response of nonlinear simulation with the PSSs is compared, and superiority of the BA-PID-PSS is established in terms of settling time and performance indices. In this section, the eigenvalue comparison is to be carried out for SMIB power system with nominal operating conditions for all controllers in Table 2. The power system, programmed in MATLAB

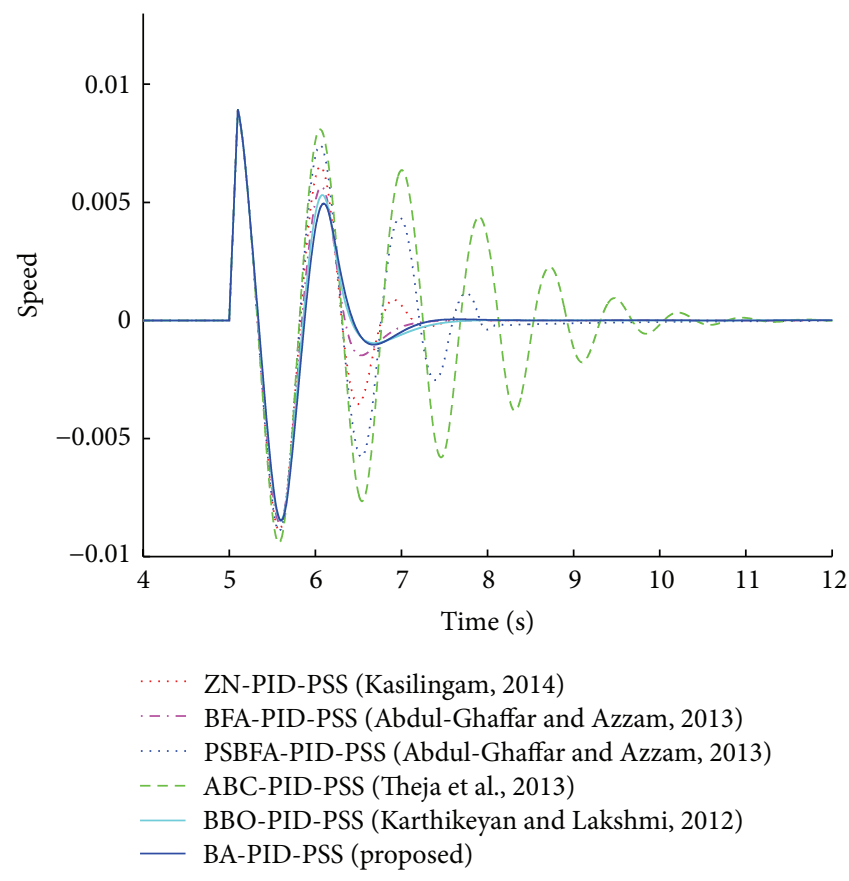

FIGURE 9: Speed response comparison of ZN [25], BFA [23], PSBFA [23], ABC [26], BBO [27], and BA (proposed) based PID type PSS for SMIB power system with nominal operating conditions.

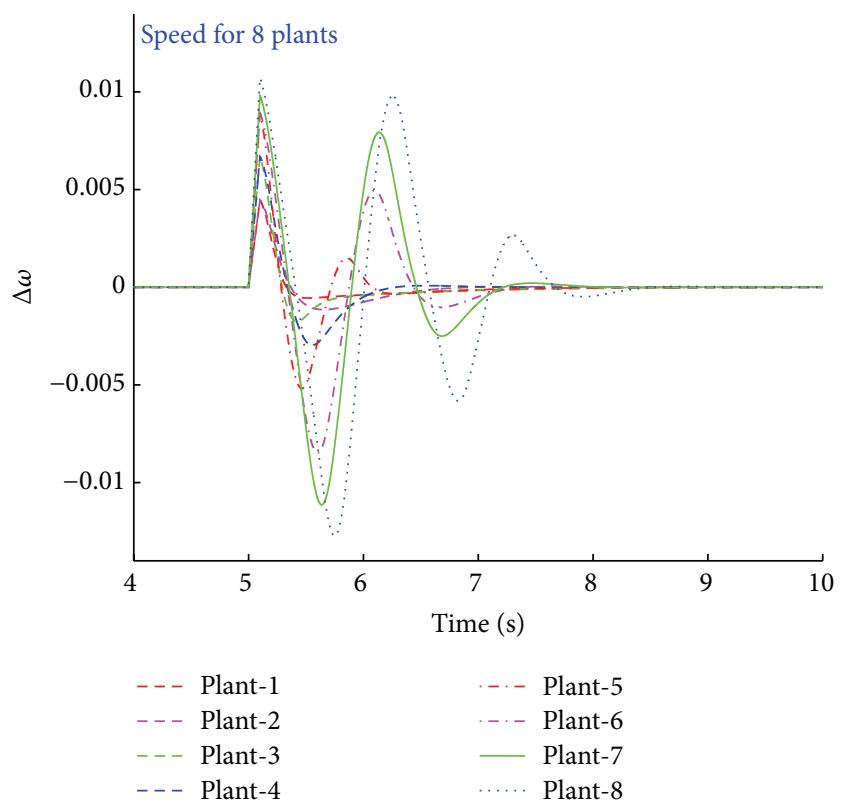

FIGURE 10: Speed response using BA-PID-PSS with eight nonlinear plant conditions.

Software (m-file and Simulink), is run to determine $A, B$, $C$, and $D$ matrices using linmod command of the software. To determine eigenvalue of the system eig function of software is used. The resulting eigenvalues have only real parts (negative) with damping ratio as 1.0 and, therefore, are not of interest. The complex conjugate eigenvalues are responsible for oscillations of the system response and are enlisted in 


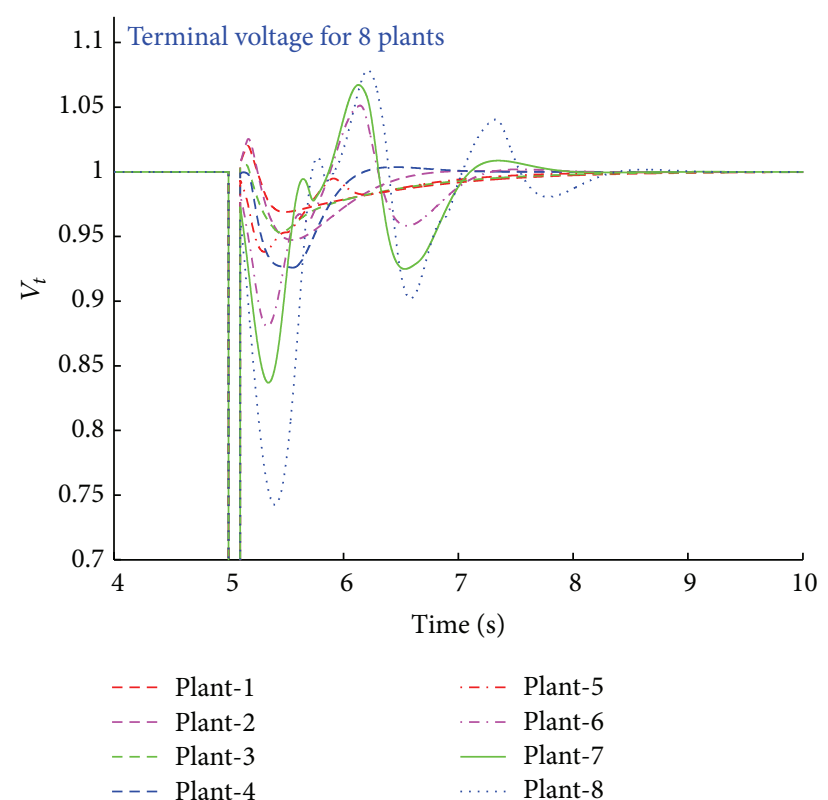

FIGURE 11: Terminal voltage response using BA-PID-PSS with eight nonlinear plant conditions.

TABLE 3: Performance indices based comparison of speed response with PID-PSS reported in literature and BA-PID-PSS on SMIB system.

\begin{tabular}{lccc}
\hline Controller & ITAE & IAE & ISE \\
\hline GA-PID-PSS [19] & 0.0541 & 0.0088 & $4.1610 E-05$ \\
HS-PID-PSS [19] & 0.0490 & 0.0081 & $3.9365 E-05$ \\
BFA-PID-PSS [22] & 0.0668 & 0.0103 & $4.4853 E-05$ \\
RCGA-PID-PSS [24] & 0.0382 & 0.0067 & $3.5710 E-05$ \\
ZN-PID-PSS [25] & 0.0441 & 0.0076 & $4.2167 E-05$ \\
BFA-PID-PSS [23] & 0.0382 & 0.0067 & $3.6350 E-05$ \\
PSBFA-PID-PSS [23] & 0.0675 & 0.0108 & $5.6106 E-05$ \\
ABC-PID-PSS [26] & 0.1120 & 0.0165 & $8.6844 E-05$ \\
BBO-PID-PSS [27] & 0.0378 & 0.0066 & $3.5029 E-05$ \\
BA-PID-PSO & 0.0371 & 0.0065 & $3.4499 E-05$ \\
\hline
\end{tabular}

Table 4. As per literature, the desirable damping ratio of the system should be more than 0.1 . On critical examination of the 3 rd column of Table 4, the maximum damping ratio of the system is associated with the proposed controller as 0.125400 , proving its superiority among the other controllers.

4.1.6. Robust Operation over 231 Plants. The line diagram of the SMIB power system is shown in Figure 1 with the configuration of system modes like without PSS, with comparing PID-PSS reported in literature (Table 2) and the transmission line connection to infinite-bus with fault location. The value of active power $\left(P_{g 0}\right)$ and the transmission line reactance $\left(X_{l}\right)$ is kept varying to formulate 231 plants of SMIB power system within the range given in (12). The value of active power is changed from 0.4 to 1.4 and reactance from 0.2 to 0.7 in the step size of 0.05 in both cases resulting in 21 and
TABLE 4: EMOs having least damping factor for SMIB system with nominal operating conditions with PID-PSS reported in literature and BA-PID-PSS.

\begin{tabular}{lccc}
\hline Controller & Eigenvalue & Dam. factor & Freq. \\
\hline GA-PID-PSS [19] & $-0.24386 \pm 6.9823 i$ & 0.034905 & 1.1113 \\
HS-PID-PSS [19] & $-0.82746 \pm 8.8267 i$ & 0.093336 & 1.4048 \\
BFA-PID-PSS [22] & $-0.85728 \pm 9.3378 i$ & 0.091423 & 1.4862 \\
RCGA-PID-PSS [24] & $-0.8924 \pm 11.083 i$ & 0.080258 & 1.764 \\
ZN-PID-PSS [25] & $-0.8741 \pm 9.1725 i$ & 0.094865 & 1.4599 \\
BFA-PID-PSS [23] & $-0.94423 \pm 9.8075 i$ & 0.095833 & 1.5609 \\
PSBFA-PID-PSS [23] & $0.86605 \pm 19.065 i$ & -0.045381 & 3.0342 \\
ABC-PID-PSS [26] & $-0.67126 \pm 8.7627 i$ & 0.076381 & 1.3946 \\
BBO-PID-PSS [27] & $-0.77408 \pm 11.198 i$ & 0.068964 & 1.7822 \\
BA-PID-PSS & $-1.0733 \pm 8.4915 i$ & 0.125400 & 1.3515 \\
\hline
\end{tabular}

11 combinations, respectively, and thus constituting 231 plant conditions of SMIB power system

$$
\begin{aligned}
& 0.4 \leq P_{g 0} \leq 1.4 \\
& 0.2 \leq X_{l} \leq 0.7
\end{aligned}
$$

The operating point of power system covered as above encompasses all practical operating conditions, constituting a set of 231 different plants (operating conditions). In previous section, the nonlinear simulation is carried out and the superiority of the proposed BA-PID-PSS is proved. In this section, the robust performance of SMIB is to be checked for 231 plant conditions (covering very heavy loading conditions) by eigenvalue plot. The eigenvalue plots for 231 plants (as defined by (12)) are drawn for all controllers in Table 2 including proposed BA-PID-PSS, and the robust operation of the proposed controller is to be established. In this section, the eigenvalues of the system are not expected to be in d-shape sector or wedge-shaped sector with the proposed BA-PIDPSS, because the objective function is a time domain based minimization of ISE of the speed error signal. Therefore, the robustness would be checked by having all eigenvalues for the wide operating conditions in the left half of s-plane (LHS). The eigenvalues of the system without PSS are sharing 146 EMOs in the right-hand side (RHS) of s-plane. The SMIB system equipped with different PID based PSS (as in Table 2) is simulated to get eigenvalue plot on s-plane. The EMOs lying in RHS are enlisted in Table 5. It is not possible to show eigenvalue plot on s-plane of system with all controllers as in Table 2 because of space limitation. The eigenvalue plot of system with GA-PID-PSS and proposed BA-PID-PSS is shown in Figures 12 and 13. It can be seen that the EMOs in RHS of s-plane with BA-PID-PSS are zero proving robust operation over the wide range of operating conditions of the power system.

\subsection{Two-Area 4-Machine 10-Bus Power System}

4.2.1. Simulation Plant Creation. The two-area four-machine ten-bus power system is considered as in [13]. The creations of system models based on operating conditions are enlisted 
TABLE 5: Detail of the number of EMOs in RHS of s-plane for SMIB power system with 231 plant configurations.

\begin{tabular}{lc}
\hline Controller & EMOs \\
\hline GA-PID-PSS [19] & 138 \\
HS-PID-PSS [19] & 4 \\
BFA-PID-PSS [22] & 6 \\
RCGA-PID-PSS [24] & 20 \\
ZN-PID-PSS [25] & 4 \\
BFA-PID-PSS [23] & 6 \\
PSBFA-PID-PSS [23] & 276 \\
ABC-PID-PSS [26] & 16 \\
BBO-PID-PSS [27] & 30 \\
BA-PID-PSS & $\mathrm{Nil}$ \\
\hline
\end{tabular}

TABLE 6: Four plant configurations of two-area four-machine tenbus power system based on active power, reactive load distribution, and bus structure [35].

\begin{tabular}{lccc}
\hline PS model & Active power & Active load & Fault \\
\hline Plant-1 & {$[7.0,7.0,7.2172,7.0]$} & $\begin{array}{l}11.59+j 2.12 \\
15.75+j 2.88\end{array}$ & Bus-3 \\
\hline Plant-2 & {$[7,7,7.2172,7]$} & $\begin{array}{l}11.59+j 2.12 \\
15.75+j 2.88\end{array}$ & \multirow{2}{*}{ Bus-4 } \\
\hline Plant-3 & {$[7.2,7.1,7.0,6.9]$} & $11.59+j 2.12$ & \multirow{2}{*}{ Bus-5 } \\
\hline \multirow{2}{*}{ Plant-4 } & {$[7.2,7.1,7.0,6.9]$} & $11.59+j 2.12$ & \multirow{2}{*}{ Bus-6 } \\
\hline Plant-5 & {$[7.2,7.1,7.0,6.9]$} & $11.99+j 2.12$ & \multirow{2}{*}{ Bus-7 } \\
\hline Plant-6 & {$[7.1,6.9,7.5,6.5]$} & $11.19+j 2.12$ & \multirow{2}{*}{ Bus-8 } \\
\hline Plant-7 & {$[7.1,6.9,7.5,6.5]$} & $11.19+j 2.12$ & \multirow{2}{*}{ Bus-9 } \\
\hline Plant-8 & {$[5,8,6.2172,8]$} & $11.59+j 2.12$ & \multirow{2}{*}{ Bus-10 } \\
\hline
\end{tabular}

in Table 6. It includes Plant-1 as base case with bus structure as in Figure 3. The last column shows the self-clearing fault location at a particular bus. The active power, active load, and the line outage are considered for creation of different experimental plants as in [35].

4.2.2. PID Based PSS Design Using Bat Algorithm. The creations of experimental plants for two-area four-machine tenbus power system are well explained in Section 4.2.1, where, in the line data, load flow data and machine data to the system are considered as in [35]. The system model referring to Plant-1 configuration as in Table 6 is equipped with PID type PSSs to all four machines (named as Gen-1 to Gen4) and subjected to PSSs design using the bat algorithm with a simple time domain based minimization of ISE as an objective function mentioned in (5). The speed signal from each generator is sensed and the value of sum of ISE of the error signal is minimized to tune PID parameters of four

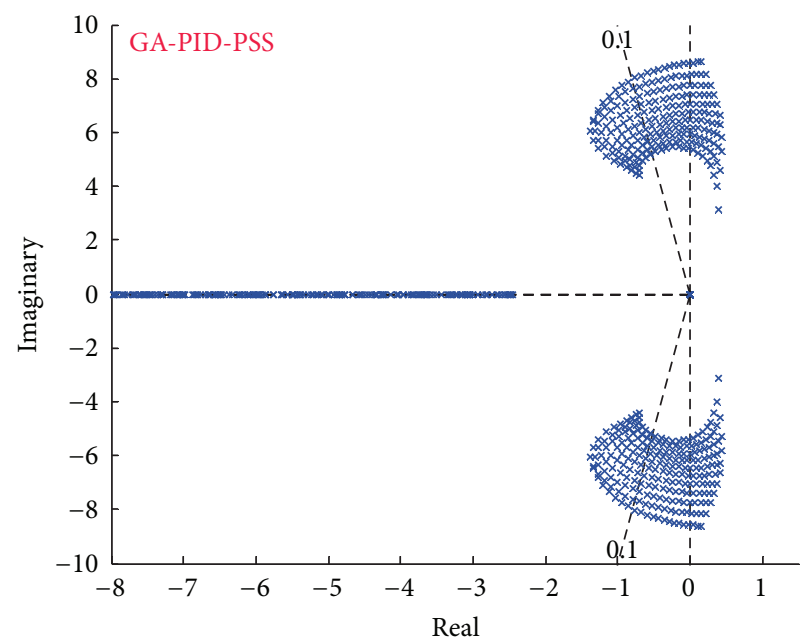

FIGURE 12: Eigenvalue plot for 231 plant conditions with GA-PIDPSS [19] on SMIB power system.

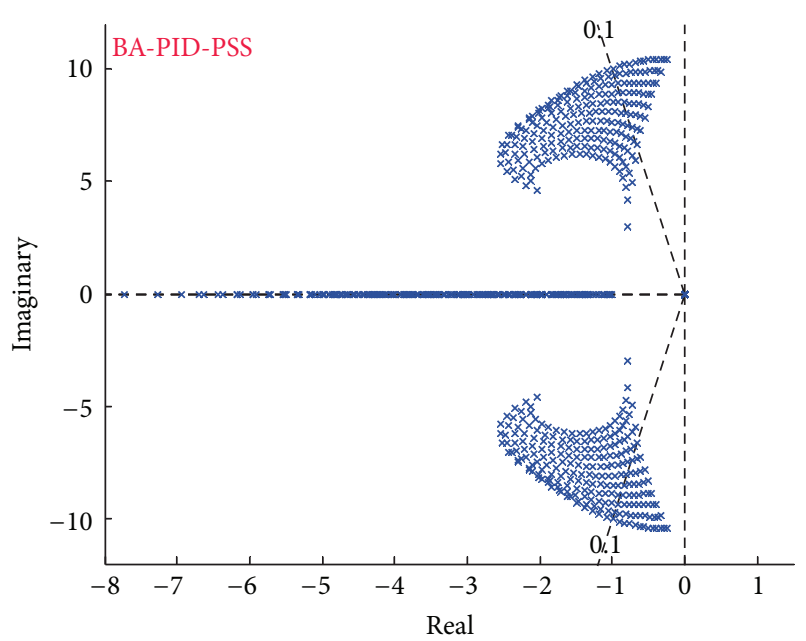

FIGURE 13: Eigenvalue plot for 231 plant conditions with proposed BA-PID-PSS on SMIB power system.

PSSs with parameter bounds as $20.0 \leq K_{j p} \leq 60.0,5.0 \leq$ $K_{j d} \leq 20.0$, and $0.01 \leq K_{j i} \leq 25.0$. By a trial-and-error method, it is found that the suitable values of loudness and pulse rate for PID-PSS optimization using the bat algorithm are as $A=0.9$ and $r=0.1$. The termination criterion of the tuning process is considered as the maximum number of iterations and set as 200. The parameter bounds are selected by using a trial-and-error method; therefore, several attempts are required. The optimized PID based PSSs parameters are shown in Table 7 to compare with that reported in literature. The behaviour of BA during optimization in terms of fitness function is shown in Figure 14. The plot of PID parameters with iteration counts for four generators (Gen-1-Gen-4) is shown in Figures 15, 16, and 17 for $K_{p}, K_{d}$, and $K_{i}$, respectively. Clearly, the 90 iterations are sufficient to have constant value of fitness function.

In literature, the PID type controller design for fourmachine power system is only designed using iterative linear 


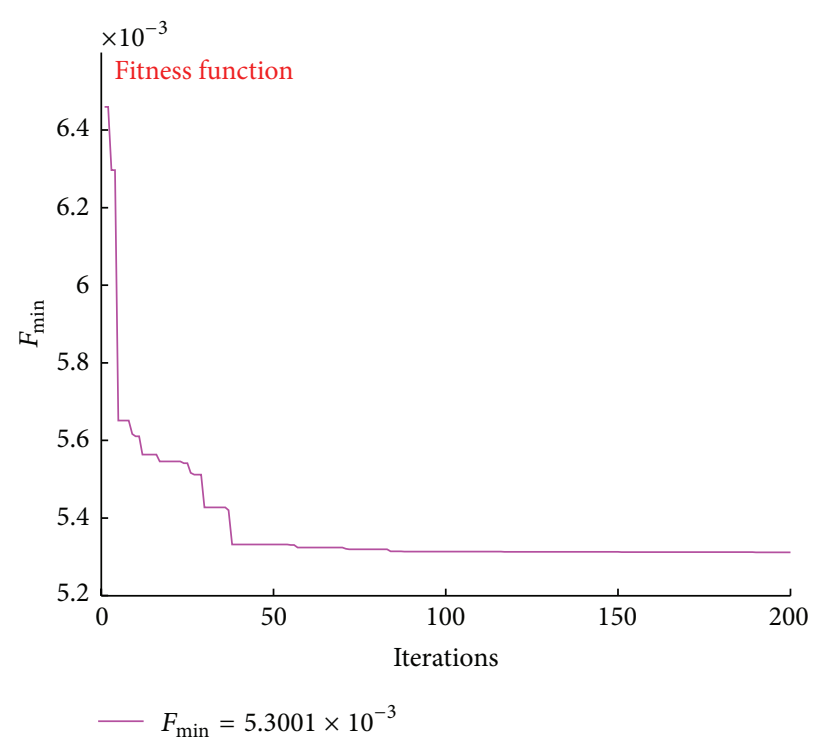

FIGURE 14: Fitness function variation with iteration in tuning of PID-PSS using bat algorithm for 4-machine 39-bus power system.

TABLE 7: Comparison of PID based PSS parameters for 4-machine 10-bus power system.

\begin{tabular}{lcccc}
\hline Controller & Geners. & $K_{p}$ & $K_{d}$ & $K_{i}$ \\
\hline \multirow{4}{*}{ ILMI-PID-PSS [18] } & Gen-1 & 47.311 & -0.0267 & 53.077 \\
& Gen-2 & 39.426 & 0.06970 & 139.84 \\
& Gen-3 & 23.285 & -0.0312 & -17.477 \\
& Gen-4 & 21.689 & -0.0796 & 54.573 \\
\hline \multirow{4}{*}{ BA-PID-PSS } & Gen-1 & 60.000 & 5.2592 & 25.000 \\
& Gen-2 & 59.990 & 19.989 & 24.999 \\
& Gen-3 & 59.998 & 10.479 & 25.000 \\
& Gen-4 & 54.270 & 5.0000 & 25.000 \\
\hline
\end{tabular}

matrix inequality (ILMI) [18], and the parameters of four PID type PSS parameters connected to four machines are enlisted in Table 7.

4.2.3. Speed Response Analysis. A Simulink based block diagram, including all the nonlinear blocks, is generated $[1,35]$. The speed signals of the machines are considered as output and the initial value of the speed is taken as zero. The output signals of CPSSs are added to $V_{\text {ref }}$ via limiters. It is used to damp out the small signal disturbances via modulating the generator excitation. The output must be limited to preventing the PSS acting to counter action of AVR. Different operating points are taken as the different plants for both systems. The limits of PSS outputs are taken as \pm 0.05 . In decentralized PSSs, to activate the proposed controller at same instant, proper synchronization signal is required to be sent to all machines. All PSSs can be applied simultaneously to the respective machines for both power system models.
The CPSSs designed in previous section are connected to the system. Each plant condition as listed in Table 6 is considered with fault locations. The disturbance is considered as self-clearing fault at different buses at 1.0 second and cleared after 0.05 seconds. The simulation and eigenvalue analysis with eight plant conditions without PSS are described and found that none of the generators of plants are showing stable operation and, therefore, not needed to compare to the simulation results $[13,35]$.

In this section, the speed response with proposed BAPID-PSS is compared to the response of the system with only controller available in literature, that is, ILMI-PID-PSS [18] and conventional PSSs designed in [41]. The parametric comparison of PID based PSS is made in Table 6. The speed response of the four-machine system of Plant-1 configuration with CPSSs [41], ILMI-PID-PSSs [18], and BA-PID-PSSs (proposed) for all four generators is compared with the system response in Figures 18-21. It can be clearly said that the speed response of Plant-1 with the use of BA-PID-PSS is greatly improved. To have the robust performance study of the proposed BA-PID-PSS, the system configuration (as eight plants) is simulated for eight times and the speed responses for each generator (Gen-1 to Gen-4) are presented in Figures $22-25$. The system response with proposed controller gives a stable response for each generator with each plant configuration.

To have a complete comparison of the system performance over a wide range of operating conditions and with different system configuration, the speed responses of system with CPSSs [41], ILMI-PID-PSSs [18], and BA-PID-PSSs (proposed) controllers are compared. Since the system plants are eight and controllers are three, therefore, the system is simulated for 24 times and each time the performance's indices (ITAE, IAE, and ISE) are recorded in Table 8. On comparison of PIs in these tables, the minimum value is 


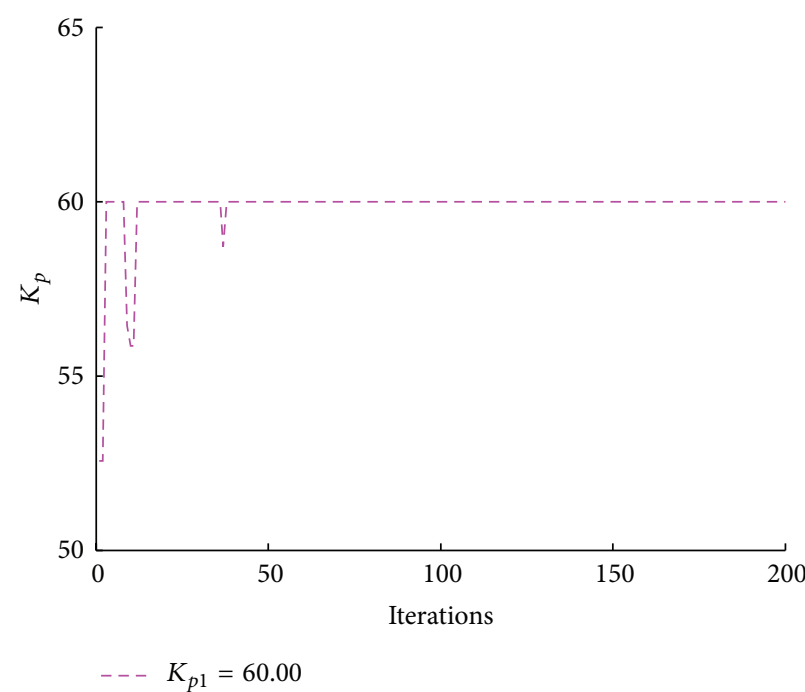

(a) Gen-1

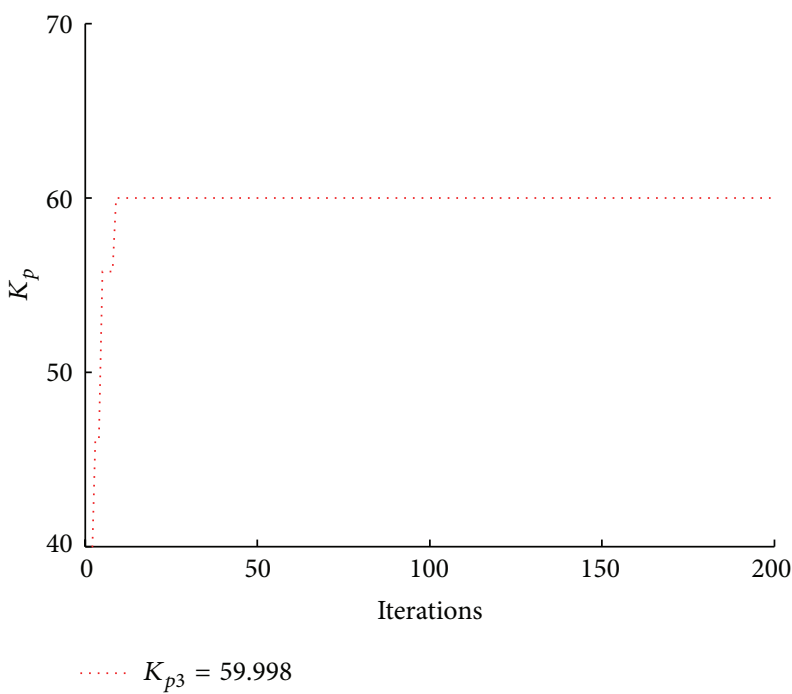

(c) Gen-3

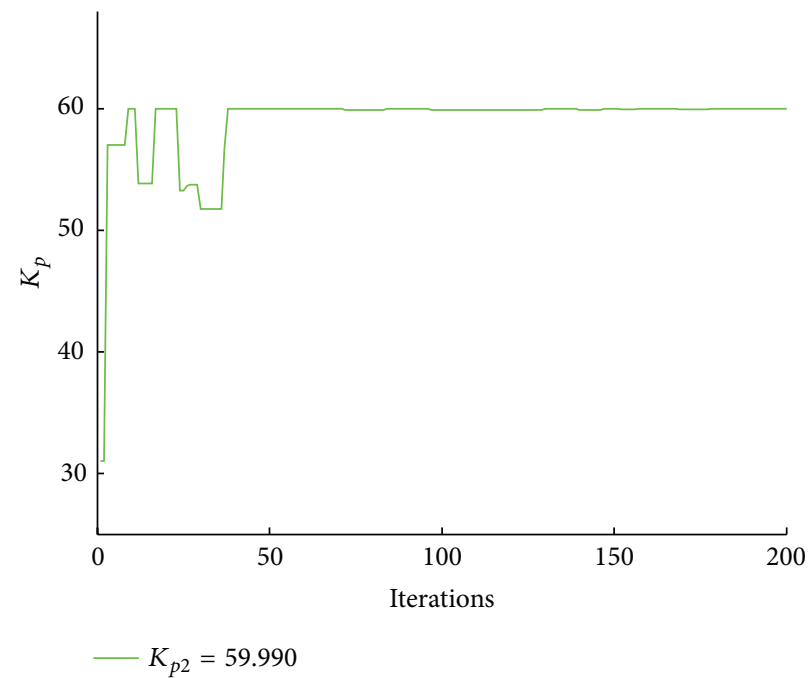

(b) Gen-2

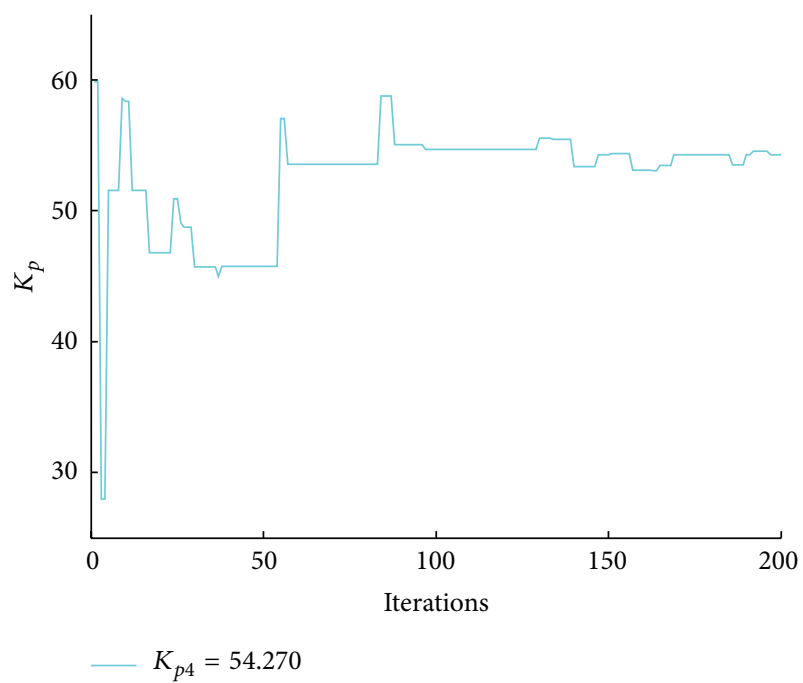

(d) Gen-4

FIGURE 15: Plot of PID-PSS parameters $\left(K_{p}\right)$ with iteration count using bat algorithm for 4-machine 10-bus power system.

attained by the proposed BA-PID-PSS for all plant conditions, proving its superior performance over the CPSSs [41] and ILMI-PID-PSSs [18].

4.2.4. Eigenvalue Analysis. In previous section, the speed response and its performance indices analysis are carried out and proved the superior performance of the proposed BA-PID-PSS over the comparing controllers. In this section, the eigenvalue analysis is carried with the system for Plant1 configuration when equipped with CPSSs [33], ILMI-PIDPSSs [15], and BA-PID-PSSs (proposed) controllers. The electromechanical modes with least value of damping ratio are recorded for each generator as in Table 9. The eigenvalue analysis reveals that the damping ratio with BA-PID-PSS is improved as compared to other controllers (CPSSs [41], ILMI-PID-PSSs [18]).

\subsection{IEEE 10-Machine 39-Bus Power System}

4.3.1. Simulation Plant Creation. The IEEE 39-bus power system is configured with different sets of active power and active load connected to the system shown in Figure 3. It has 10 generators and 19 loads connected as in [35]. The active power assigned to Plant-1 (base case) is as in [5.519816, 10.0, $6.5,5.08,6.32,6.5,5.6,5.4,8.3,2.5]$. The load assigned to Plant-1 (base case) for bus numbers $[1,2,13,14,17,18,21,23$, $24,25,26,27,28,29,30,32,35,36,38]=[(0.092+j 0.046)$, $(11.04+j 2.5),(3.22+j 0.024),(5.0+j 1.84),(2.3380+j 0.8400)$, $(5.22+j 1.76),(2.74+j 1.15),(2.745+j 0.8466),(3.086+j 0.922)$, $(2.24+j 0.472),(1.39+j 0.17),(2.81+j 0.755),(2.06+j 0.276)$, $(2.835+j 0.269),(6.28+j 1.030),(.075+j 0.88),(3.20+j 1.53)$, $(3.294+j 0.323)$, and $(1.58+j 0.30)]$. To generate 8-plant configurations, the different sets of active power of generators and active load are considered. These system plants are shown 


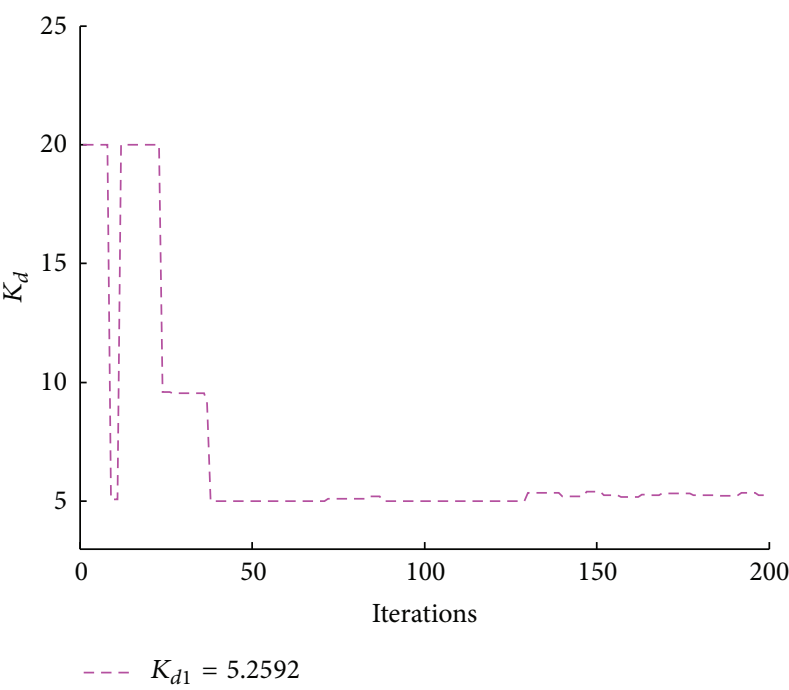

(a) Gen-1

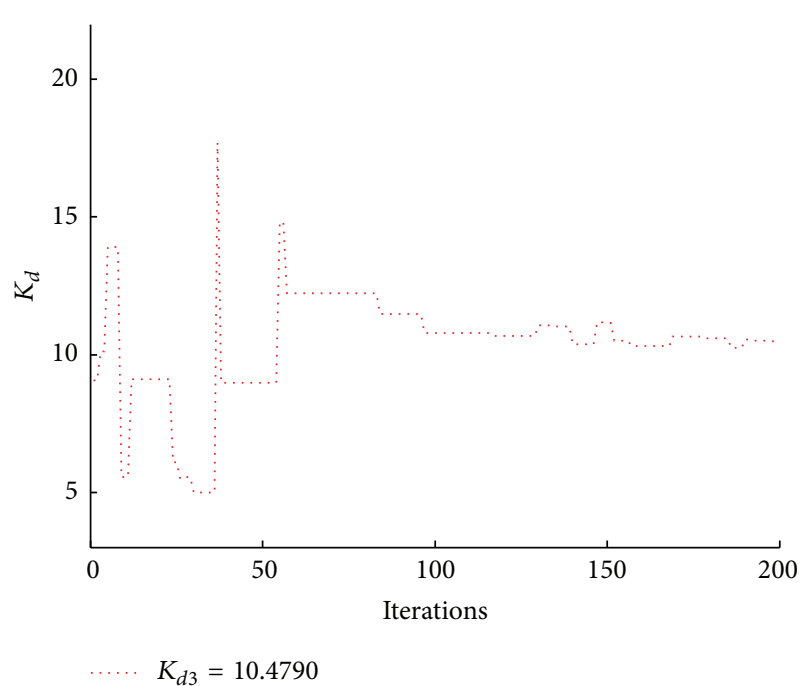

(c) Gen-3

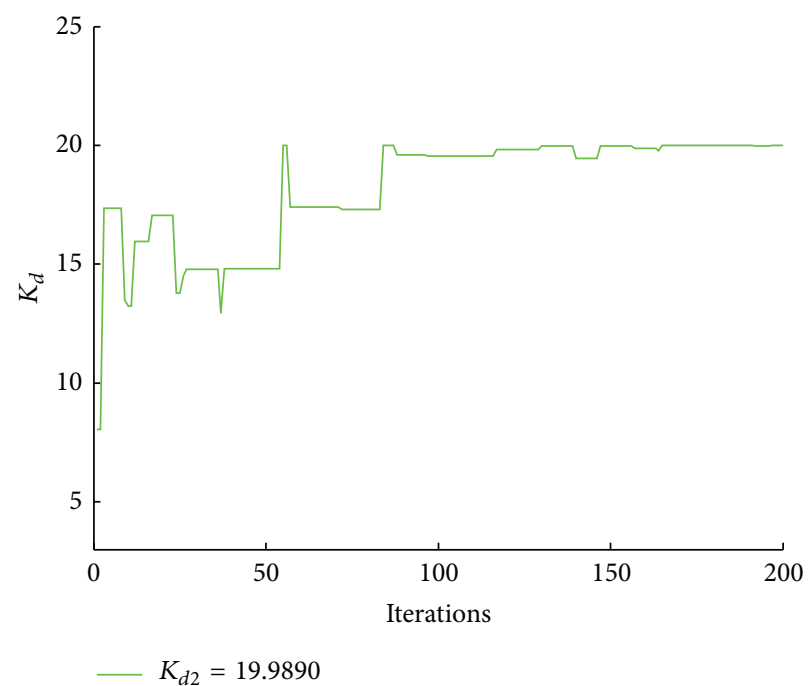

(b) Gen-2

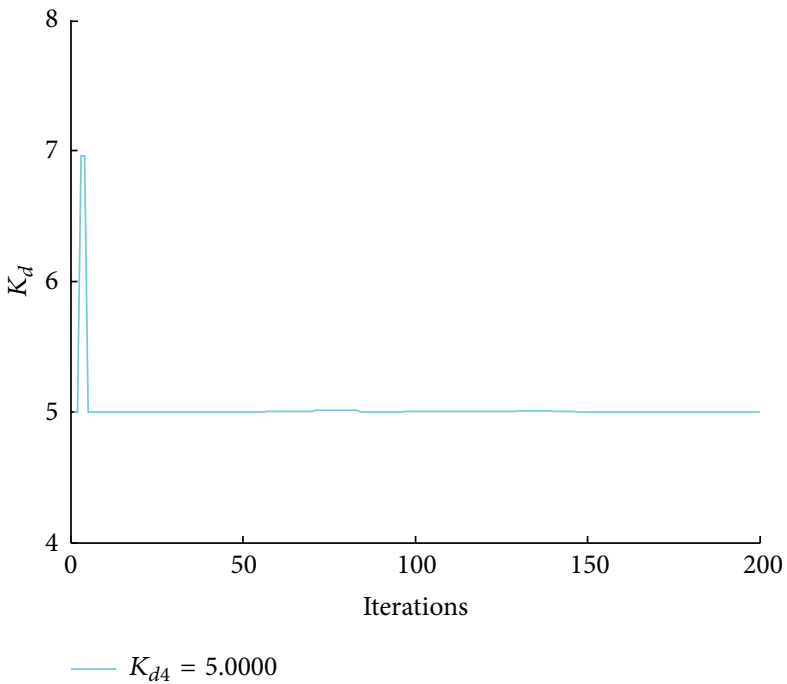

(d) Gen-4

FIGURE 16: Plot of PID-PSS parameters $\left(K_{d}\right)$ with iteration count using bat algorithm for 4-machine 10-bus power system.

in Table 10. The last column of Table 10 refers to the selfclearing fault at a bus for nonlinear behaviour of system.

4.3.2. PID Based PSS Design Using Bat Algorithm. The creation of experimental plants for IEEE New England tenmachine thirty-nine-bus power system is well explained in Section 4.3.1, where, in the machine data, load flow data, transformer data, and line data for the system are considered as in $[1,35]$. The system model, referring to Plant- 1 configuration as in Table 10, is equipped with PID type PSSs to all nine machines (named as Gen-1 to Gen-9) except Gen-10, which is considered as the slack and subjected to PSSs design using bat algorithm as described in Section 3 with parameter bounds as $20.0 \leq K_{j p} \leq 60.0,20.0 \leq K_{j d} \leq 60.0$, and $0.01 \leq K_{j i} \leq 25.0$. With the initializing parameters as above of bat algorithm, the power system is simulated to tune the
CPSSs parameters for an iteration count as 200. The problem of optimization is considered with minimization of ISE of speed response with parameter bounds. The fitness function variation for 200 iterations is shown in Figure 26, wherein the fitness value becomes constant at around 180 iterations. The optimal set PID controller values are enlisted in Table 11.

4.3.3. Speed Response Analysis. The IEEE New England tenmachine thirty-nine-bus power system is subjected to simulation with the designed BA-PID-PSS and other comparing controllers. The disturbance is considered as self-clearing at different buses at 1.0 second and cleared after 0.05 seconds. The simulation response and eigenvalue analysis with eight plant conditions without PSS are unstable and, therefore, not needed to compare to the forthcoming simulation results. 


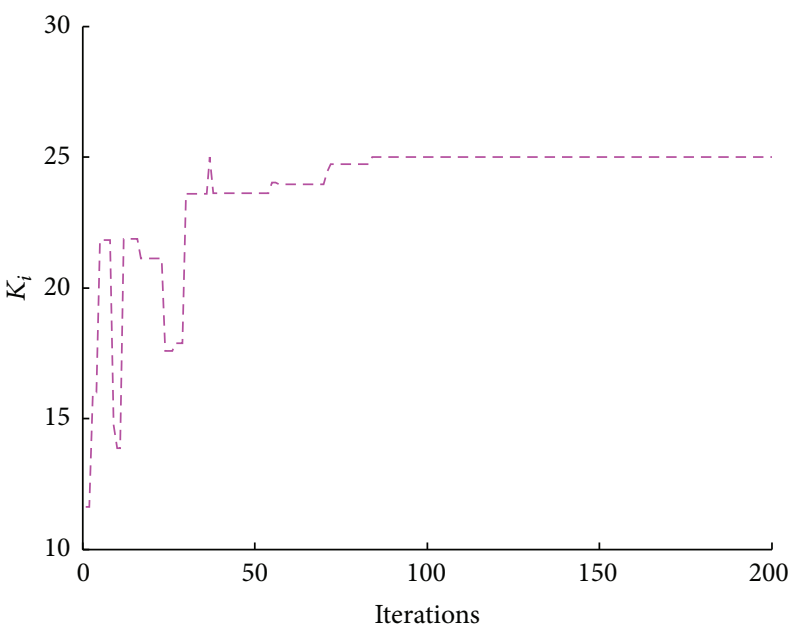

$---K_{i 1}=25.000$

(a) Gen-1

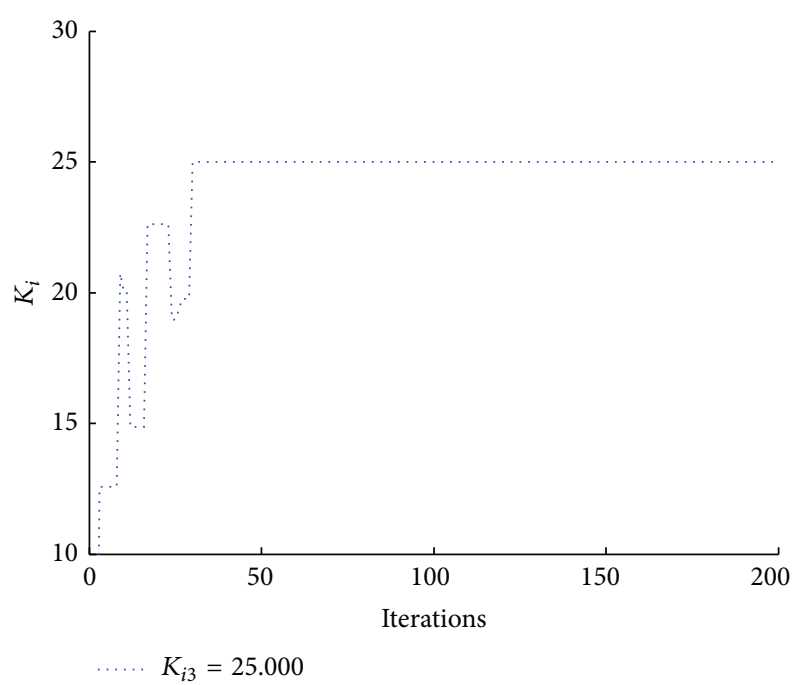

(c) Gen-3

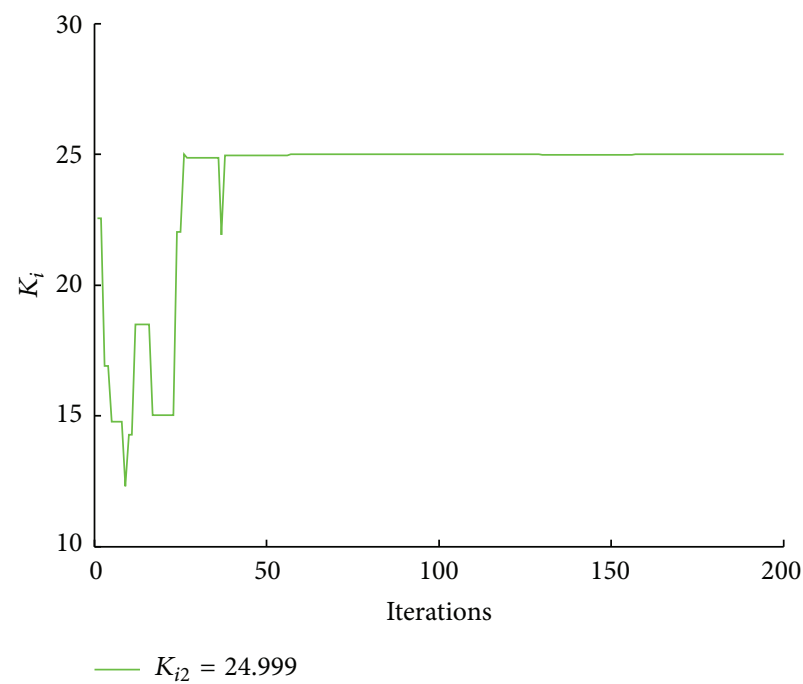

(b) Gen-2

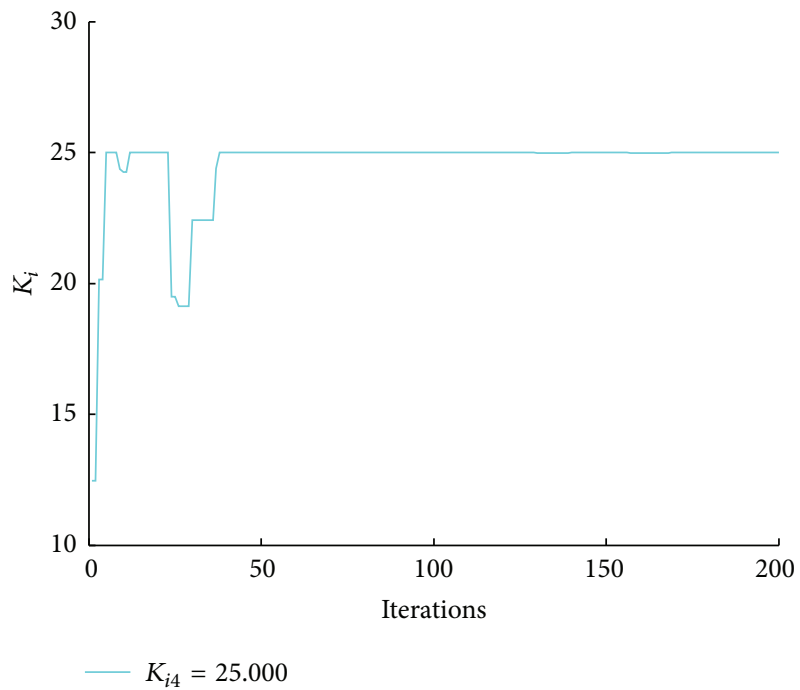

(d) Gen-4

FIGURE 17: Plot of PID-PSS parameters $\left(K_{i}\right)$ with iteration count using bat algorithm for 4-machine 10-bus power system.

A Simulink based block diagram, including all the nonlinear blocks, is generated $[1,35]$. The speed signals of the machines are considered as output and the initial value of the speed deviation is taken as zero. It is used to damp out the small signal disturbances via modulating the generator excitation. The output must be limited to preventing the PSS acting to counter action of AVR. Different operating points are taken as the different plants for the system. The limits of PSS outputs are taken as \pm 0.05 . In decentralized PSS, to activate the proposed controller at same instant, proper synchronization signal is required to be sent to all machines. All PSSs can be applied simultaneously to the respective machines for the power system.

In this section genetic algorithm (GA) [42], ant colony optimization (ACO) [43], and strength-pareto evolutionary algorithm (SPEA) [44] based CPSSs are used to compare the response with proposed BA-PID-PSS.
The simulation study with nonlinear Plant-1 configuration is carried out four times because of 3-CPSSs controllers and one proposed BA-PID-PSS. Each time the speed responses of all ten generators are recorded and plotted for Gen-1, Gen-2, Gen-5, and Gen-8 in Figures 27, 28, 29, and 30 , respectively. It is clear that the application of BA-PIDPSS improves the speed response in terms of settling time and overshoots. To check robustness of the proposed PID type PSS, the simulation is again carried out with eight plants (referring to wide range of operating conditions with different system configurations) and the speed response is recorded. The plot of speed response for Gen-1, Gen-3, Gen-5, and Gen8 with eight plants is shown in Figures 31,32, 33, and 34, respectively. It is easy to say that the system is stable for all eight plant conditions with BA-PID-PSSs.

4.3.4. PI Based Analysis. As in previous section the comparing CPSSs are GA-CPSS [42], ant colony optimization 
TABLE 8: Performance indices based comparison of speed response for Plants-1-8 of 4-machine 10-bus system with CPSS [41], ILMI-PID-PSS [18], and BA-PID-PSS (proposed).

\begin{tabular}{|c|c|c|c|c|}
\hline PS model & Controller & ITAE & IAE & ISE \\
\hline \multirow{3}{*}{ Plant-1 } & CPSS [41] & 0.0752 & 0.0251 & $6.0598 E-05$ \\
\hline & ILMI-PID-PSS [18] & 0.0469 & 0.0194 & $5.4053 E-05$ \\
\hline & BA-PID-PSS (prop.) & 0.0201 & 0.0112 & $3.2318 E-05$ \\
\hline \multirow{3}{*}{ Plant-2 } & CPSS [41] & 0.1042 & 0.0351 & $1.0987 E-04$ \\
\hline & ILMI-PID-PSS [18] & 0.0808 & 0.0317 & $1.2326 E-04$ \\
\hline & BA-PID-PSS (prop.) & 0.0434 & 0.0214 & $8.4797 E-05$ \\
\hline \multirow{3}{*}{ Plant-3 } & CPSS [41] & 0.0442 & 0.0150 & $1.9582 E-05$ \\
\hline & ILMI-PID-PSS [18] & 0.0363 & 0.0130 & $1.9048 E-05$ \\
\hline & BA-PID-PSS (prop.) & 0.0134 & 0.0068 & $9.0028 E-06$ \\
\hline \multirow{3}{*}{ Plant-4 } & CPSS [41] & 0.0384 & 0.0137 & $1.5681 E-05$ \\
\hline & ILMI-PID-PSS [18] & 0.0398 & 0.0141 & $1.9356 E-05$ \\
\hline & BA-PID-PSS (prop.) & 0.0119 & 0.0058 & $6.9224 E-06$ \\
\hline \multirow{3}{*}{ Plant-5 } & CPSS [41] & 0.0460 & 0.0151 & $1.8749 E-05$ \\
\hline & ILMI-PID-PSS [18] & 0.0228 & 0.0096 & $1.3308 E-05$ \\
\hline & BA-PID-PSS (prop.) & 0.0112 & 0.0057 & $7.2262 E-06$ \\
\hline \multirow{3}{*}{ Plant-6 } & CPSS [41] & 0.0379 & 0.0128 & $1.3858 E-05$ \\
\hline & ILMI-PID-PSS [18] & 0.0183 & 0.0084 & $1.0583 E-05$ \\
\hline & BA-PID-PSS (prop.) & 0.0093 & 0.0052 & $6.2257 E-06$ \\
\hline \multirow{3}{*}{ Plant-7 } & CPSS [41] & 0.0282 & 0.0103 & $7.0640 E-06$ \\
\hline & ILMI-PID-PSS [18] & 0.0123 & 0.0054 & $2.9837 E-06$ \\
\hline & BA-PID-PSS (prop.) & 0.0119 & 0.0049 & $2.7070 E-06$ \\
\hline \multirow{3}{*}{ Plant-8 } & CPSS [41] & 0.0257 & 0.0096 & $7.0855 E-06$ \\
\hline & ILMI-PID-PSS [18] & 0.0195 & 0.0084 & $7.8744 E-06$ \\
\hline & BA-PID-PSS (prop.) & 0.0061 & 0.0030 & $1.9644 E-06$ \\
\hline
\end{tabular}

TABLE 9: EMOs with least damping factor for Plant-1 with CPSS [41], ILMI-PID-PSS [18], and proposed BA-PID-PSS.

\begin{tabular}{lccc}
\hline Controller & Eigenvalue & Dam. factor & Freq. \\
\hline & $-0.63261 \pm 7.241 i$ & 0.087033 & 1.1524 \\
CPSS [41] & $-0.69844 \pm 7.8906 i$ & 0.08817 & 1.2558 \\
& $-0.47113 \pm 4.1411 i$ & 0.11304 & 0.65907 \\
& $-9.1938 \pm 19.344 i$ & 0.42927 & 3.0786 \\
\hline & $-0.45173 \pm 11.077 i$ & 0.040747 & 1.763 \\
ILMI-PID-PSS [18] & $-0.87931 \pm 8.3531 i$ & 0.10469 & 1.3294 \\
& $-2.0076 \pm 4.629 i$ & 0.39789 & 0.73673 \\
& $-7.9106 \pm 17.918 i$ & 0.40387 & 2.8518 \\
\hline & $-0.78886 \pm 12.602 i$ & 0.062476 & 2.0056 \\
BA-PID-PSS (prop.) & $-1.2327 \pm 11.513 i$ & 0.10647 & 1.8323 \\
& $-5.6764 \pm 18.295 i$ & 0.29633 & 2.9118 \\
& $-6.3388 \pm 15.563 i$ & 0.37722 & 2.4769 \\
\hline
\end{tabular}

(ACO) [43], and strength-pareto evolutionary algorithm (SPEA) [44]; therefore, the performance of proposed BAPID-PSS should also be compared to system responses for all eight plant configurations of Table 10 with these CPSSs. The simulation is carried out for 32 times and each time the performance indices (ITAE, IAE, and ISE) of speed response are recorded for simulation time as 30 seconds. The values of these PIs are enlisted in Table 12 for all considered plant conditions. The comparatively lower values of PIs with BAPID-PSS prove its superiority over other controllers [42-44].

\section{Conclusions}

In this paper, the application of the bat algorithm is used to tune the parameters of PID based PSS for three systems such as single-machine infinite-bus (SMIB) power system, 


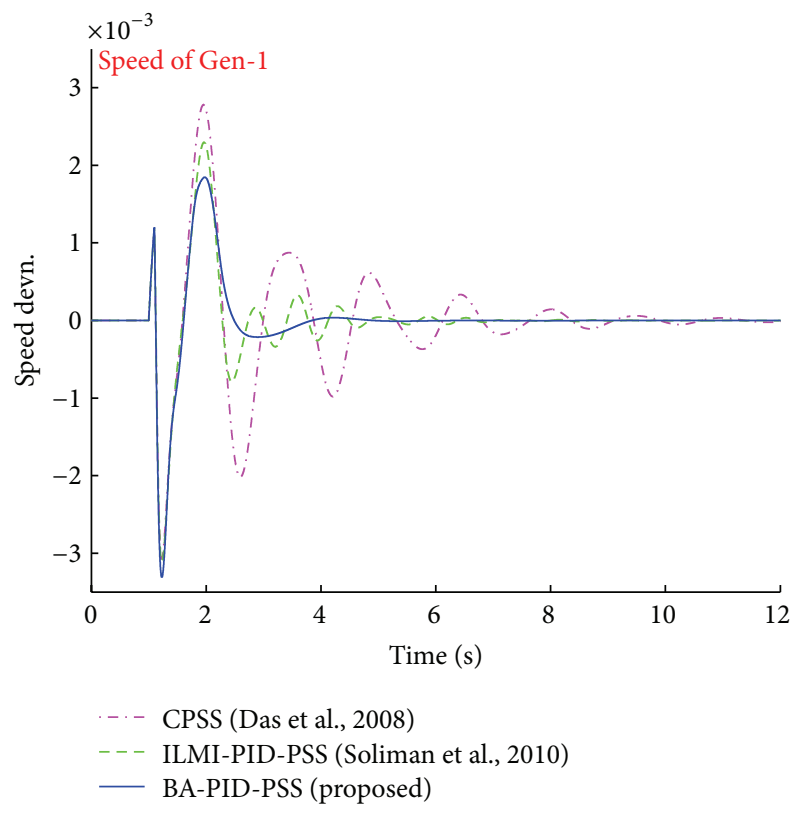

FIGURE 18: Speed response comparison for Gen-1 of Plant-1 with CPSS [41], ILMI-PID-PSS [18], and proposed BA-PID-PSS.

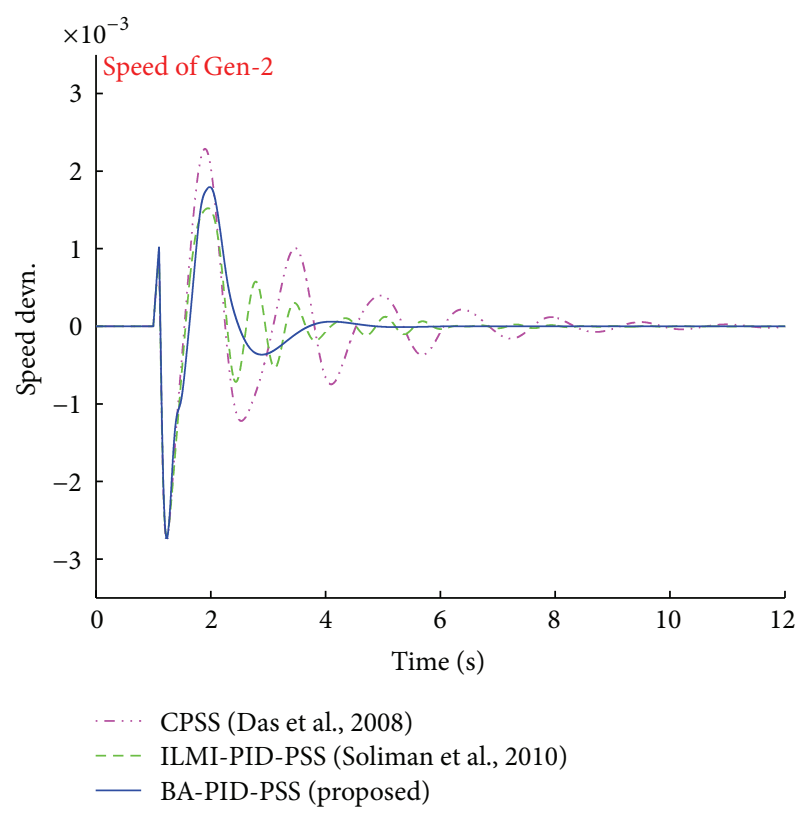

FIGURE 19: Speed response comparison for Gen-2 of Plant-1 with CPSS [41], ILMI-PID-PSS [18], and proposed BA-PID-PSS.

two-area four-machine ten-bus power system, and IEEE New England ten-machine thirty-nine-bus power systems.

The designed BA tuned PID based PSS is connected to SMIB power system and simulation study is carried out with the eight nonlinear plant conditions; the speed response is compared to the PID based PSSs reported in $[19,22-27]$. The speed response of system with BA-PIDPSS is compared to that reported in literature and found with reduced overshoot and settling time. The lesser value

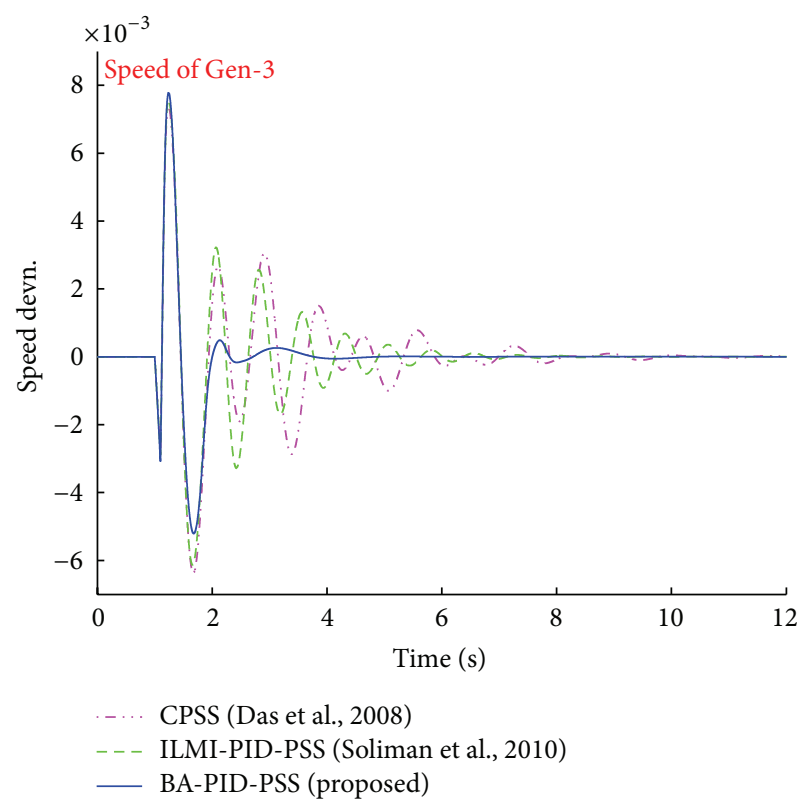

FIGURE 20: Speed response comparison for Gen-3 of Plant-1 with CPSS [41], ILMI-PID-PSS [18], and proposed BA-PID-PSS.

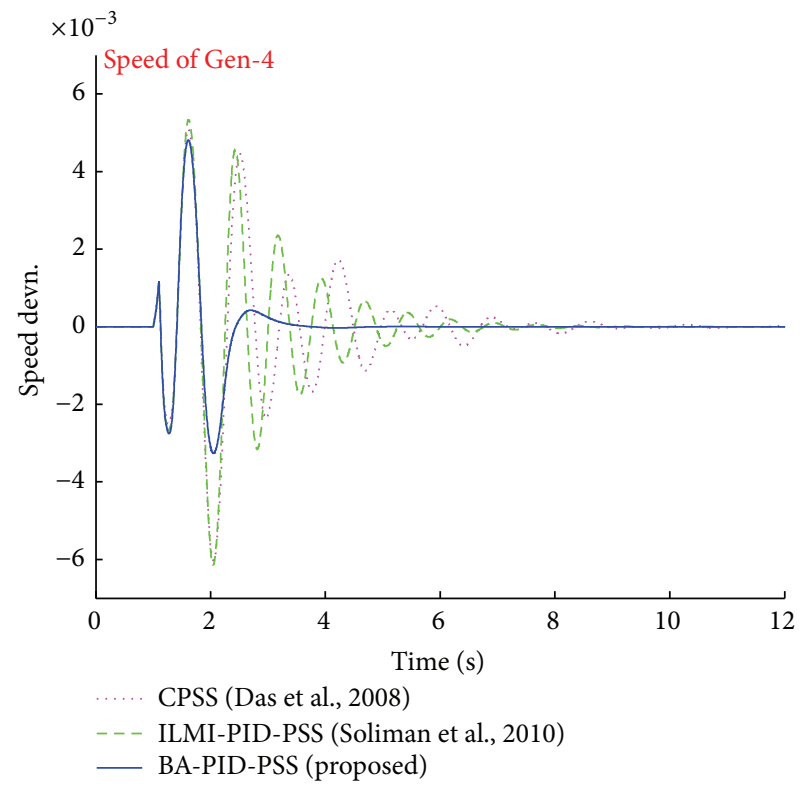

FIGURE 21: Speed response comparison for Gen-4 of Plant-1 with CPSS [41], ILMI-PID-PSS [18], and proposed BA-PID-PSS.

of performance indices of speed response with the proposed controller proves the superior performance as compared to that with the controllers in [19, 22-27]. The eigenvalue analysis gives information of damping ratio as better with BAPID-PSS as compared to others. The eigenvalue plot for 231 plant conditions determines the robustness and applicability of the proposed controller.

The bat algorithm based BA-PID-PSSs are connected to four-machine power system in decentralized manner and simulation study is carried out with the eight nonlinear plant 


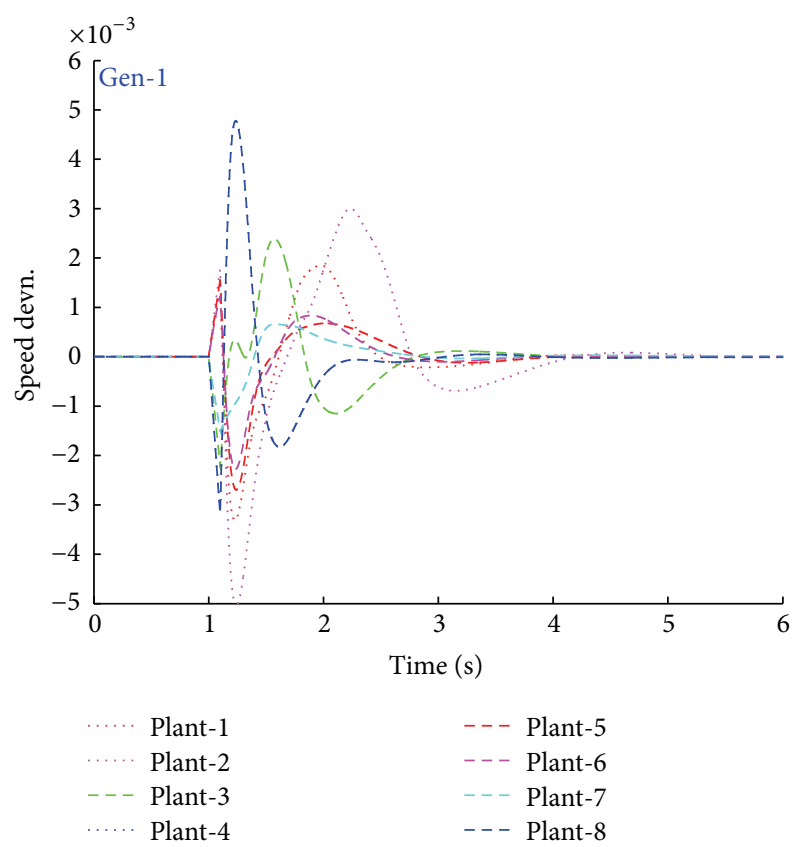

FIGURE 22: Speed response comparison for Gen-1 of all plants with proposed BA-PID-PSS.

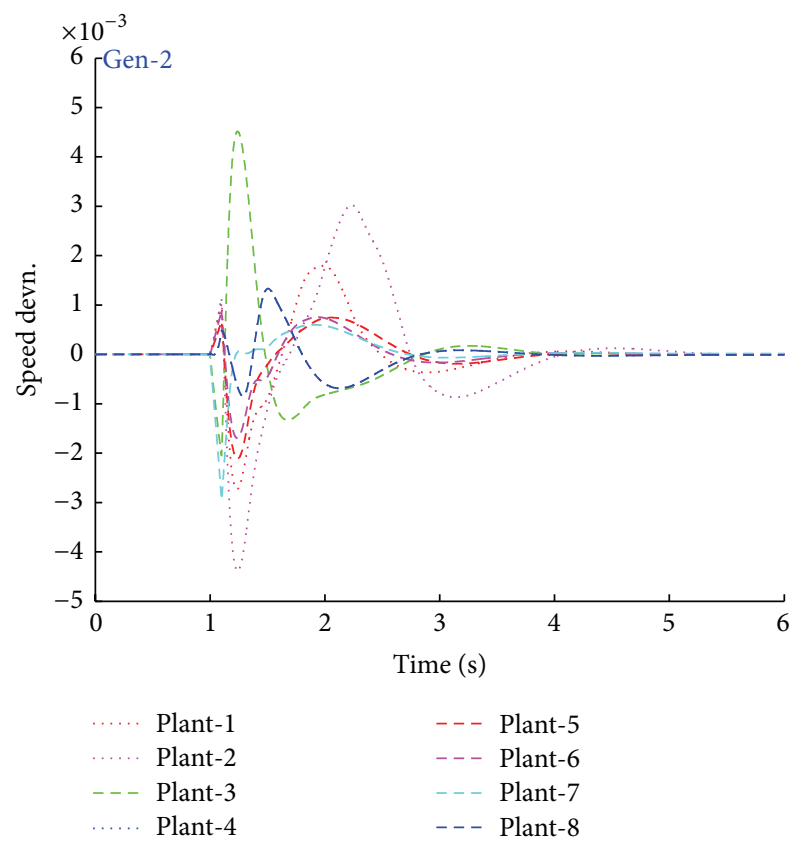

FIGURE 23: Speed response comparison for Gen-2 of all plants with proposed BA-PID-PSS.

conditions. The speed response of system with proposed BAPID-PSSs is compared to that with CPSSs [41] and ILMIPID-PSSs [18]. The nonlinear simulation results and the eigenvalue analysis reveal the superiority of the BA-PIDPSS as compared to that of others with reduced settling time and overshoot in terms of performance indices. The superiority of the proposed controller proved by performance indices (relatively lesser value) and the eigenvalue analysis

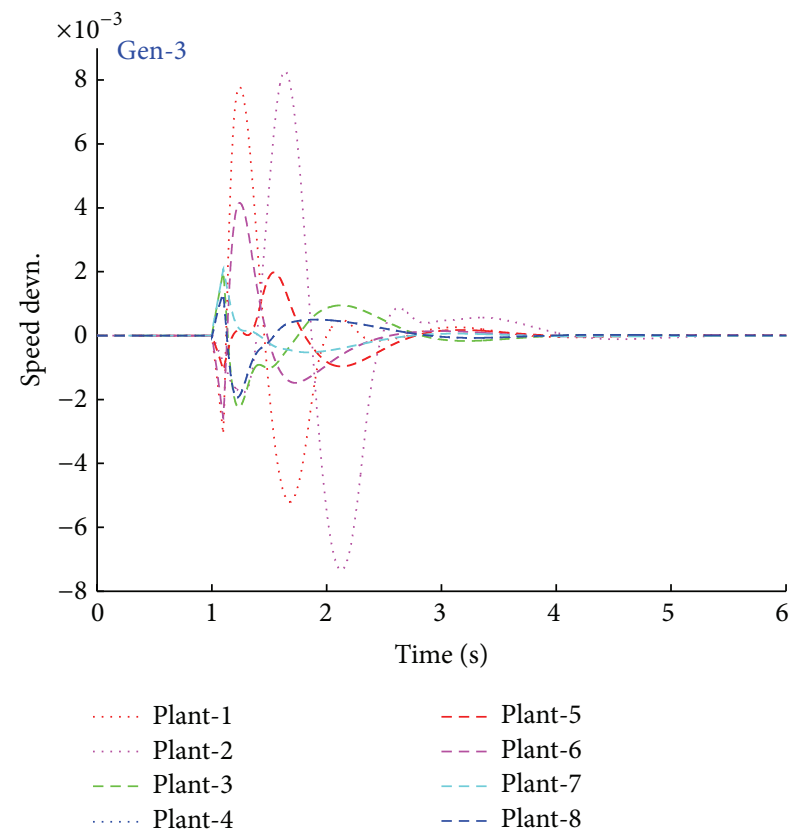

FIGURE 24: Speed response comparison for Gen-3 of all plants with proposed BA-PID-PSS.

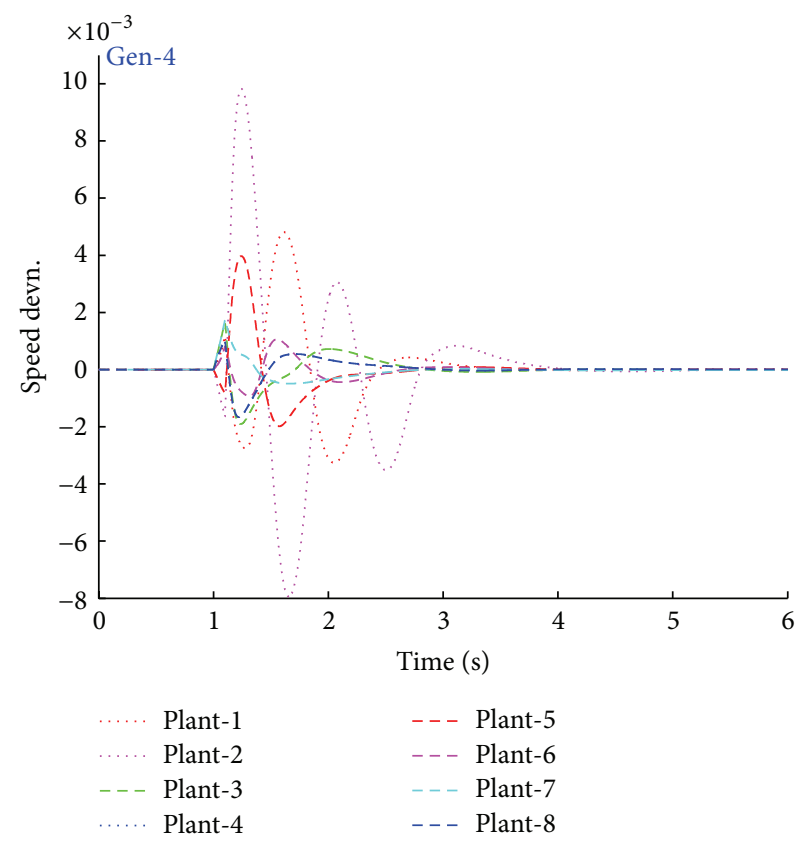

FIGURE 25: Speed response comparison for Gen-4 of all plants with proposed BA-PID-PSS.

(relatively greater value of damping ratio) over the controllers is reported in literature $[18,41]$.

The application of the bat algorithm is extended to the standard IEEE ten-machine power system network to tune the PID based PSS parameters with minimization of ISE. The nonlinear time domain simulation results of the system with proposed BA-PID-PSS and that with CPSSs as reported in literature [42-44] are compared. The speed response of the 


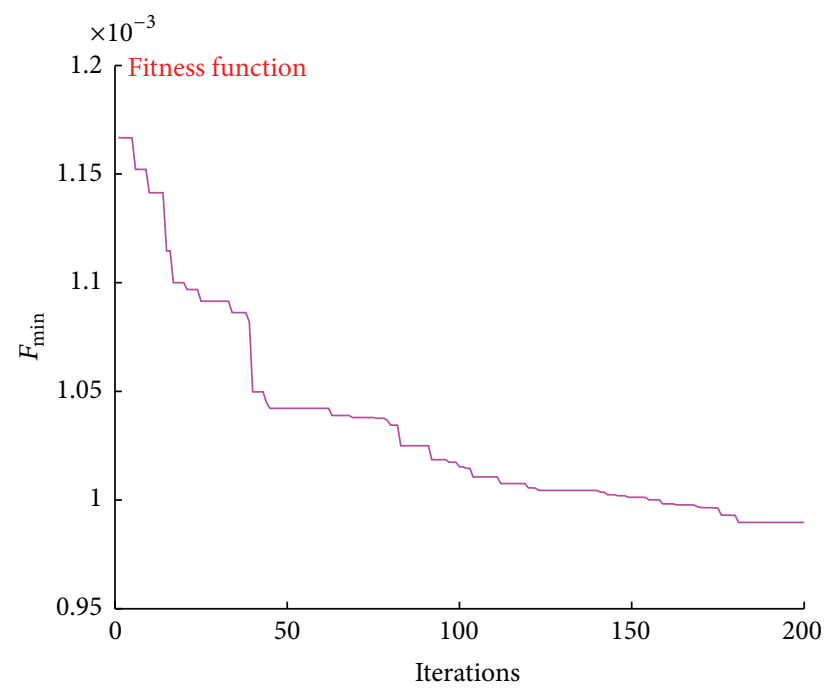

FIgURE 26: Fitness function variation with iteration during PID parameter optimization in 10-machine 39-bus power system.

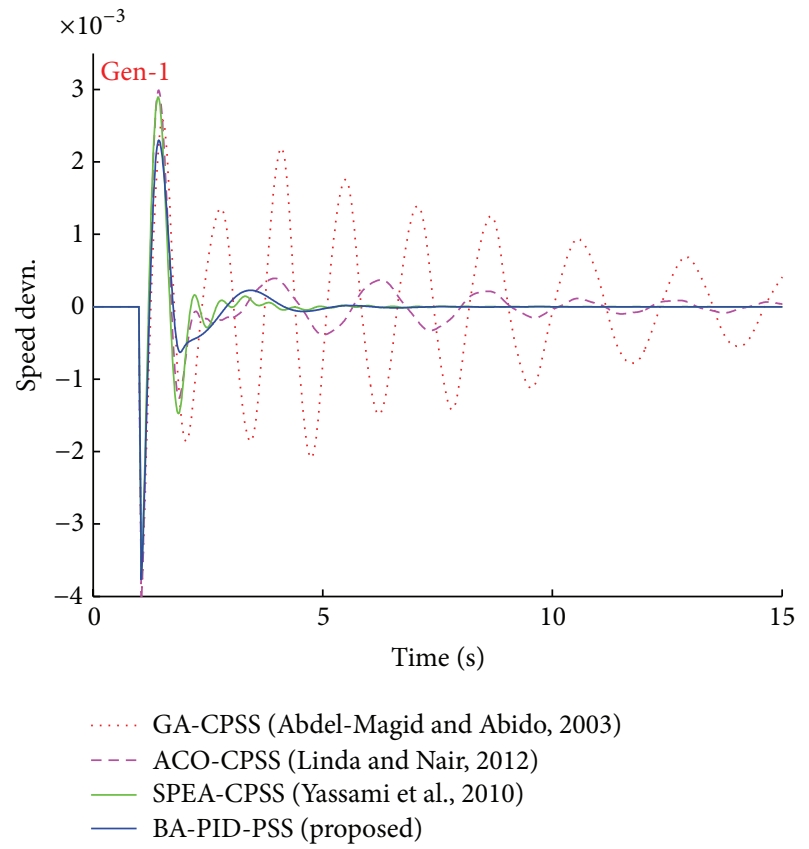

FIgURE 27: Speed response for Gen-1 of Plant-1 on 10-machine 39bus power system with GA-CPSS [42], ACO-CPSS [43], SPEA-CPSS [44], and BA-PID-PSS.

system with proposed controller can stabilize more quickly with reduced overshoot as compared to that with others in subject. The recorded performance indices of speed response with proposed BA-PID-PSS appeared with lesser value as compared to CPSSs in [42-44]. The eigenvalue analysis proves the superiority of the performance with higher value of damping ratio as compared to that of CPSSs in [42-44].

The performance of the systems with proposed BA-PIDPSS is proved to be better as compared with PID-PSSs [19, 2227], ILMI-PID-PSS [18], and CPSSs [42-44]. However, the

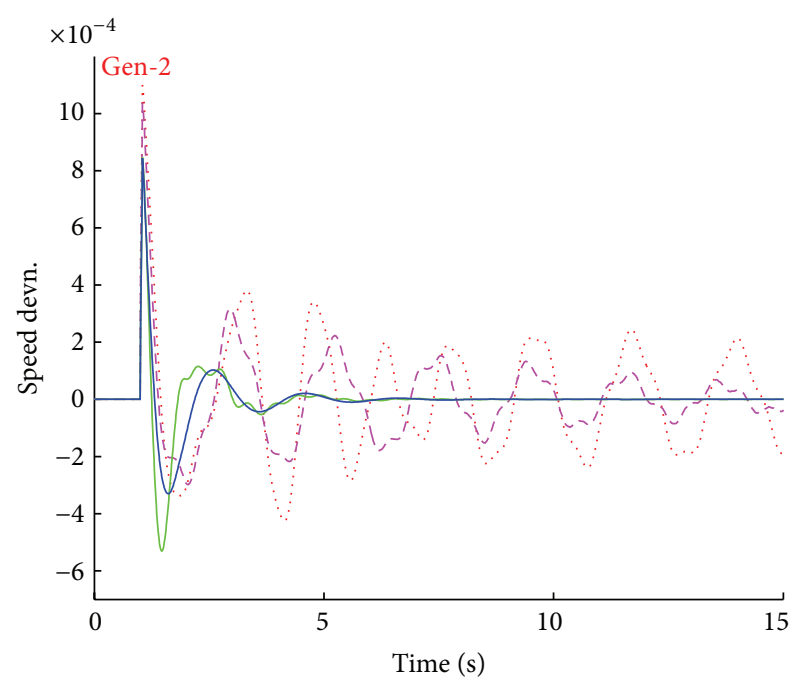

… GA-CPSS (Abdel-Magid and Abido, 2003)
--- ACO-CPSS (Linda and Nair, 2012)
- SPEA-CPSS (Yassami et al., 2010)
- BA-PID-PSS (proposed)

FIGURE 28: Speed response for Gen-2 of Plant-1 on 10-machine 39bus power system with GA-CPSS [42], ACO-CPSS [43], SPEA-CPSS [44], and BA-PID-PSS.

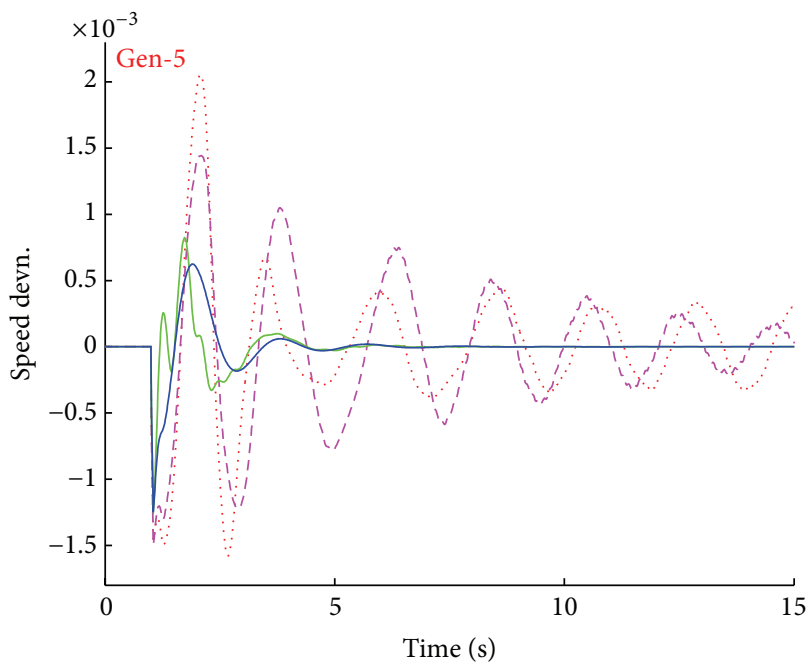

GA-CPSS (Abdel-Magid and Abido, 2003)

- - - ACO-CPSS (Linda and Nair, 2012)

_ SPEA-CPSS (Yassami et al., 2010)

BA-PID-PSS (proposed)

FIgURE 29: Speed response for Gen-5 of Plant-1 on 10-machine 39bus power system with GA-CPSS [42], ACO-CPSS [43], SPEA-CPSS [44], and BA-PID-PSS.

performance of BA-PID-PSS is not comparable to that with BA-CPSSs. It has been observed that the PID parameter tuning using an eigenvalue based objective function is unable to provide acceptable solutions; therefore, simple time domain based objective function has been used. It could be one of the 

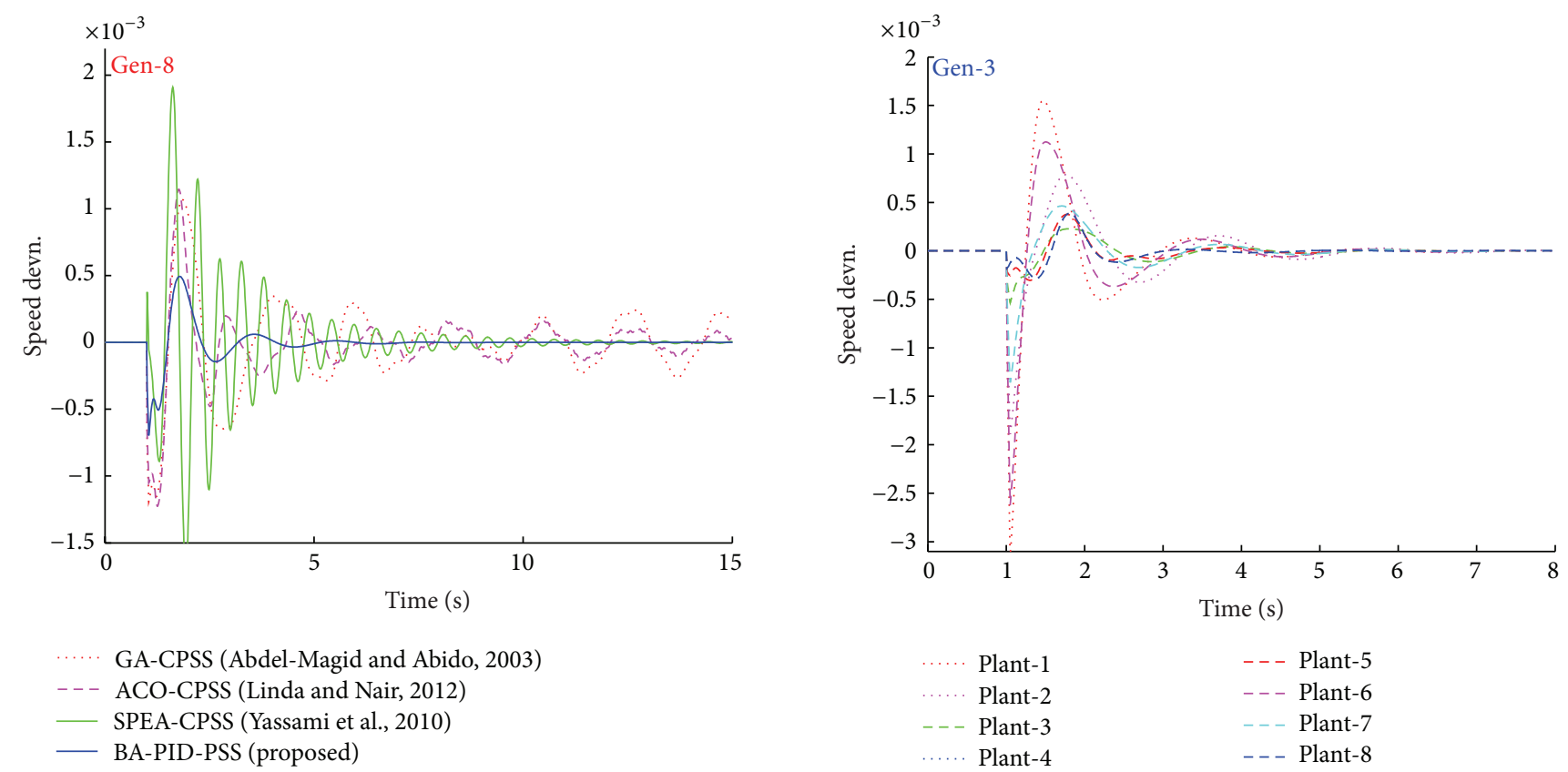

FIGURE 30: Speed response for Gen-8 of Plant-1 on 10-machine 39bus power system with GA-CPSS [42], ACO-CPSS [43], SPEA-CPSS [44], and BA-PID-PSS.

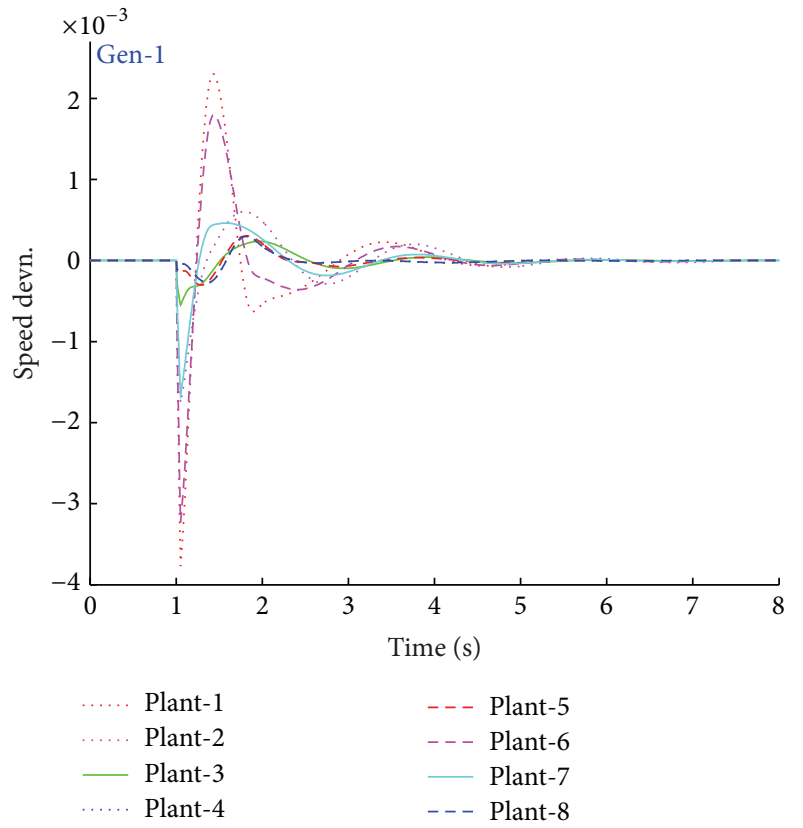

Figure 31: Speed response for Gen-1 of Plants-1-8 of 10-machine 39bus power system with proposed BA-PID-PSS.

reasons that the performance of systems with BA-PID-PSS is not more effective than that of BA-CPSSs.

\section{Competing Interests}

The authors declare that there are no competing interests regarding the publication of this paper.
Figure 32: Speed response for Gen-3 of Plants-1-8 of 10-machine 39-bus power system with proposed BA-PID-PSS.

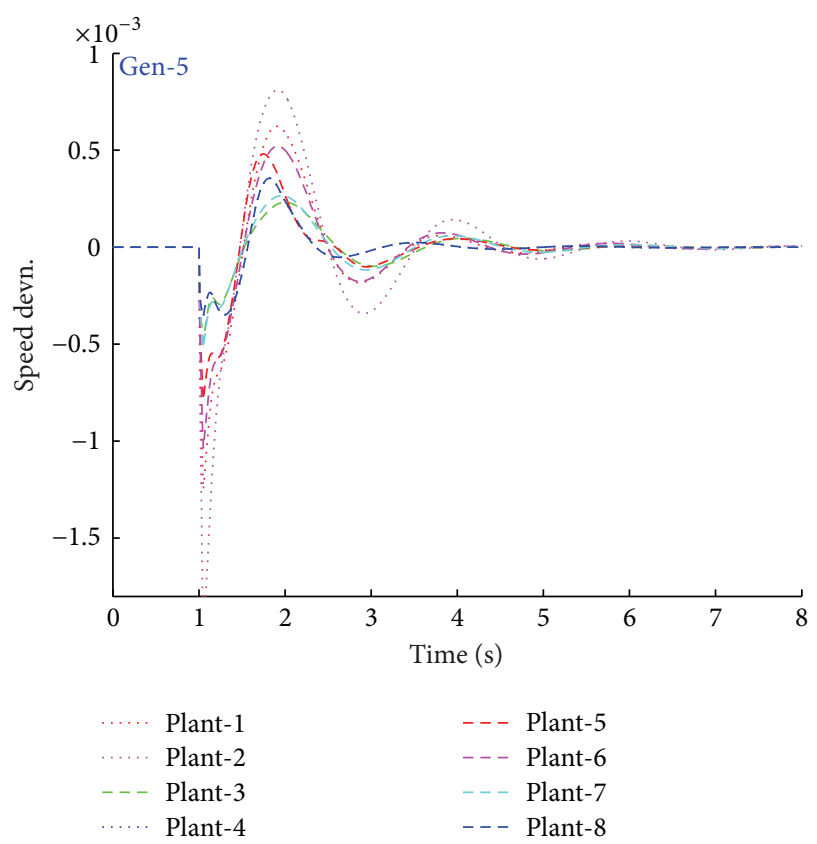

FIGURE 33: Speed response for Gen-5 of Plants-1-8 of 10-machine 39-bus power system with proposed BA-PID-PSS.

\section{Acknowledgments}

This research was supported by All India Council of Technical Education, New Delhi, India. The authors are grateful to University College of Engineering, Rajasthan Technical University, Kota, for sponsoring them under Quality Improvement Programme. D. K. Sambariya also thanks his colleagues for 


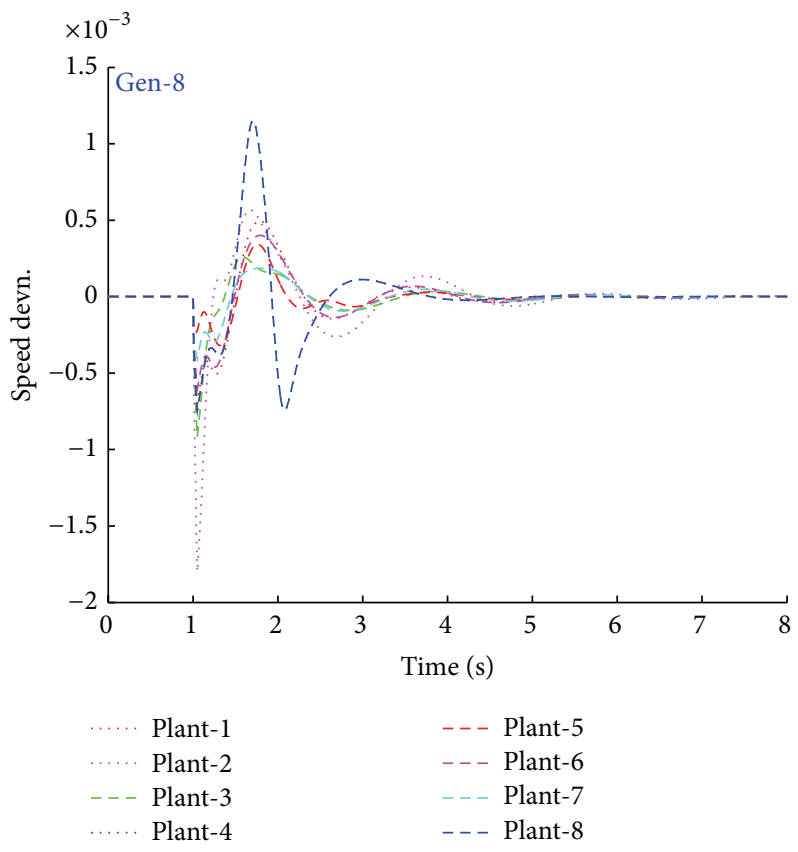

FIGURE 34: Speed response for Gen-8 of Plants-1-8 of 10-machine 39-bus power system with proposed BA-PID-PSS.

TABLE 10: Plant configuration with different operating conditions for IEEE New England 10-machine 39-bus power system [35].

\begin{tabular}{lccc}
\hline PS model & Active power $^{\mathrm{a}}$ & Active load $^{\mathrm{b}}$ & Fault at bus $^{\text {B }}$ \\
\hline Plant-1 & Base case & Base case & Bus-13 \\
Plant-2 & 3,5 & $2,13,27,28$ & Bus-11 \\
Plant-3 & $1,2,3,4$ & 17,24 & Bus-9 \\
Plant-4 & 7,8 & $27,28,30,32$ & Bus-7 \\
Plant-5 & 2,7 & $30,35,36,38$ & Bus-17 \\
Plant-6 & $1,3,9,10$ & $24-27,30,35,36$ & Bus-19 \\
Plant-7 & $1,4,5,6$ & $13,25,30,35$ & Bus-21 \\
Plant-8 & $4,5,6,7$ & $18,21,27,28,36,38$ & \\
\hline
\end{tabular}

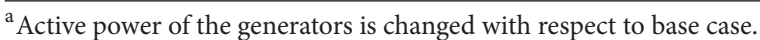

${ }^{\mathrm{b}}$ The connected load to the buses is changed with respect to base case.

TABLE 11: Optimal settings for PID based PSS parameters using bat algorithm for 10-machine 39-bus power system.

\begin{tabular}{lccc}
\hline Generators & $K_{p}$ & $K_{d}$ & $K_{i}$ \\
\hline Gen-1 & 0.69040 & 50.0000 & 6.09810 \\
Gen-2 & 49.5740 & 9.9091 & 23.9860 \\
Gen-3 & 12.2050 & 25.0880 & 25.0000 \\
Gen-4 & 50.0000 & 23.9720 & 23.9370 \\
Gen-5 & 46.7900 & 39.2490 & 24.8720 \\
Gen-6 & 50.0000 & 15.5030 & 24.9040 \\
Gen-7 & 46.8710 & 24.4330 & 24.3130 \\
Gen-8 & 18.0610 & 40.7080 & 23.7110 \\
Gen-9 & 49.5310 & 10.3740 & 22.8630 \\
\hline
\end{tabular}


TABLE 12: PI based comparison of speed response with GA-CPSS [42], ACO-CPSS [43], SPEA-CPSS [44], and proposed BA-PID-PSS for Plants-1-8 of 10-machine 39-bus power system.

\begin{tabular}{|c|c|c|c|c|}
\hline PS model & Controller & ITAE & IAE & ISE \\
\hline \multirow{4}{*}{ Plant-1 } & GA-CPSS [42] & 0.5058 & 0.0706 & $5.7778 E-05$ \\
\hline & ACO-CPSS [43] & 0.2191 & 0.0412 & $2.7658 E-05$ \\
\hline & SPEA-CPSS [44] & 0.0357 & 0.0164 & $1.3156 E-05$ \\
\hline & BA-PID-PSS (prop.) & 0.0184 & 0.0106 & $7.3609 E-06$ \\
\hline \multirow{4}{*}{ Plant-2 } & GA-CPSS [42] & 0.5465 & 0.0751 & $5.1958 E-05$ \\
\hline & ACO-CPSS [43] & 0.2893 & 0.0492 & $2.9828 E-05$ \\
\hline & SPEA-CPSS [44] & 0.0371 & 0.0175 & $1.4232 E-05$ \\
\hline & BA-PID-PSS (prop.) & 0.0245 & 0.0123 & $6.7891 E-06$ \\
\hline \multirow{4}{*}{ Plant-3 } & GA-CPSS [42] & 0.3058 & 0.0432 & $1.7065 E-05$ \\
\hline & ACO-CPSS [43] & 0.1661 & 0.0299 & $1.1854 E-05$ \\
\hline & SPEA-CPSS [44] & 0.0213 & 0.0094 & $4.0660 E-06$ \\
\hline & BA-PID-PSS (prop.) & 0.0087 & 0.0054 & $1.2355 E-06$ \\
\hline \multirow{4}{*}{ Plant-4 } & GA-CPSS [42] & 0.5308 & 0.0767 & $7.7482 E-05$ \\
\hline & ACO-CPSS [43] & 0.4977 & 0.0726 & $6.8426 E-05$ \\
\hline & SPEA-CPSS [44] & 0.0612 & 0.0242 & $3.8760 E-05$ \\
\hline & BA-PID-PSS (prop.) & 0.0119 & 0.0082 & $9.8820 E-06$ \\
\hline \multirow{4}{*}{ Plant-5 } & GA-CPSS [42] & 0.3175 & 0.0490 & $2.7905 E-05$ \\
\hline & ACO-CPSS [43] & 0.2065 & 0.0385 & $2.3980 E-05$ \\
\hline & SPEA-CPSS [44] & 0.0319 & 0.0144 & $1.1481 E-05$ \\
\hline & BA-PID-PSS (prop.) & 0.0125 & 0.0081 & $7.1431 E-06$ \\
\hline \multirow{4}{*}{ Plant-6 } & GA-CPSS [42] & 0.4491 & 0.0629 & $4.4569 E-05$ \\
\hline & ACO-CPSS [43] & 0.1884 & 0.0368 & $2.1708 E-05$ \\
\hline & SPEA-CPSS [44] & 0.0326 & 0.0144 & $9.5331 E-06$ \\
\hline & BA-PID-PSS (prop.) & 0.0169 & 0.0095 & $5.2514 E-06$ \\
\hline \multirow{4}{*}{ Plant-7 } & GA-CPSS [42] & 0.2040 & 0.0348 & $1.5736 E-05$ \\
\hline & ACO-CPSS [43] & 0.1596 & 0.0299 & $1.2726 E-05$ \\
\hline & SPEA-CPSS [44] & 0.0219 & 0.0096 & $4.1197 E-06$ \\
\hline & BA-PID-PSS (prop.) & 0.0105 & 0.0060 & $1.6533 E-06$ \\
\hline \multirow{4}{*}{ Plant-8 } & GA-CPSS [42] & 0.4115 & 0.0595 & $3.6522 E-05$ \\
\hline & ACO-CPSS [43] & 0.2160 & 0.0427 & $2.8966 E-05$ \\
\hline & SPEA-CPSS [44] & 0.0383 & 0.0172 & $1.3420 E-05$ \\
\hline & BA-PID-PSS (prop.) & 0.0219 & 0.0115 & $7.3241 E-06$ \\
\hline
\end{tabular}

sharing the responsibility at the Parent Institute during his stay at Roorkee.

\section{References}

[1] K. R. Padiyar, Power System Dynamics Stability and Control, BS Publications, Hyderabad, India, 2nd edition, 2008.

[2] M. Abido, "Robust design of power system stabilizers for multimachine power systems using differential evolution," in Computational Intelligence in Power Engineering, vol. 302 of Studies in Computational Intelligence, pp. 1-18, Springer, Berlin, Germany, 2010.

[3] M. Eslami, H. Shareef, A. Mohamed, and M. Khajehzadeh, "A hybrid pso technique for damping electro-mechanical oscillations in large power system," in Proceedings of the IEEE Student Conference on Research and Development (SCOReD '10), pp. 442-447, Kuala Lumpur, Malaysia, December 2010.
[4] D. K. Sambariya and R. Prasad, "Robust tuning of power system stabilizer for small signal stability enhancement using metaheuristic bat algorithm," International Journal of Electrical Power \& Energy Systems, vol. 61, pp. 229-238, 2014.

[5] M. Ramirez-Gonzalez and O. P. Malik, "Self-tuned power system stabilizer based on a simple fuzzy logic controller," Electric Power Components and Systems, vol. 38, no. 4, pp. 407423, 2010.

[6] D. K. Sambariya, "Power system stabilizer design using compressed rule base of fuzzy logic controller," Journal of Electrical and Electronic Engineering, vol. 3, no. 3, pp. 52-64, 2015.

[7] D. K. Sambariya, R. Gupta, and A. K. Sharma, "Fuzzy applications to single machine power system stabilizers," Journal of Theoretical and Applied Information Technology, vol. 5, no. 3, pp. 317-324, 2009.

[8] M. A. Abido and Y. L. Abdel-Magid, "Eigenvalue assignments in multimachine power systems using tabu search algorithm," Computers and Electrical Engineering, vol. 28, no. 6, pp. 527-545, 2002. 
[9] M. A. Abido and Y. L. Abdel-Magid, "Optimal design of power system stabilizers using evolutionary programming," IEEE Transactions on Energy Conversion, vol. 17, no. 4, pp. 429436, 2002.

[10] M. A. Abido, "Simulated annealing based approach to PSS and FACTS based stabilizer tuning," International Journal of Electrical Power \& Energy System, vol. 22, no. 4, pp. 247-258, 2000.

[11] S. M. Abd-Elazim and E. S. Ali, "Power system stability enhancement via bacteria foraging optimization algorithm," Arabian Journal for Science and Engineering, vol. 38, no. 3, pp. 599-611, 2013.

[12] M. A. Awadallah and H. M. Soliman, "A neuro-fuzzy adaptive power system stabilizer using genetic algorithms," Electric Power Components and Systems, vol. 37, no. 2, pp. 158-173, 2009.

[13] D. K. Sambariya and R. Prasad, "Optimal tuning of fuzzy logic power system stabilizer using harmony search algorithm," International Journal of Fuzzy Systems, vol. 17, no. 3, pp. 457-470, 2015.

[14] A. Shrivastava, M. Dubey, and Y. Kumar, "Design of interactive artificial bee colony based multiband power system stabilizers in multimachine power system," in Proceedings of the IEEE International Conference on Control, Automation, Robotics and Embedded Systems (CARE '13), pp. 1-6, IEEE, Jabalpur, India, December 2013.

[15] A. M. El-Zonkoly, A. A. Khalil, and N. M. Ahmied, "Optimal tunning of lead-lag and fuzzy logic power system stabilizers using particle swarm optimization," Expert Systems with Applications, vol. 36, no. 2, pp. 2097-2106, 2009.

[16] S. M. Abd-Elazim and E. S. Ali, "A hybrid particle swarm optimization and bacterial foraging for optimal power system stabilizers design," International Journal of Electrical Power \& Energy Systems, vol. 46, no. 1, pp. 334-341, 2013.

[17] D. K. Sambariya and R. Prasad, "Design of PSS for SMIB system using robust fast output sampling feedback technique," in Proceedings of the 7th International Conference on Intelligent Systems and Control (ISCO '13), pp. 166-171, IEEE, Tamil Nadu, India, January 2013.

[18] M. Soliman, A. L. Elshafei, F. Bendary, and W. Mansour, "Robust decentralized PID-based power system stabilizer design using an ILMI approach," Electric Power Systems Research, vol. 80, no. 12, pp. 1488-1497, 2010.

[19] K. A. Hameed and S. Palani, "Robust design of power system stabilizer using harmony search algorithm," Automatika, vol. 55, no. 2, pp. 1-8, 2014.

[20] P. Bera, D. Das, and T. K. Basu, "Design of P-I-D power system stabilizer for multimachine system," in Proceedings of the 1st IEEE India Annual Conference (INDICON '04), pp. 446-450, IEEE, Kharagpur, India, December 2004.

[21] D. K. Sambariya and R. Prasad, "Design of robust PID power system stabilizer for multimachine power system using HS algorithm," American Journal of Electrical and Electronic Engineering, vol. 3, no. 3, pp. 75-82, 2015.

[22] K. Abdul Hameed and S. Palani, "Robust design of power system stabilizer using bacterial foraging algorithm," Archives of Electrical Engineering, vol. 62, no. 1, pp. 141-152, 2013.

[23] E. A. E. H. I. Abdul-Ghaffar and M. Azzam, "Design of pid controller for power system stabilization using hybrid particle swarm-bacteria foraging optimization," WSEAS Transactions on Power Systems, vol. 8, no. 1, pp. 12-23, 2013.

[24] S. Duman and A. Öztürk, "Robust design of PID controller for power system stabilization by using real coded genetic algorithm," International Review of Electrical Engineering, vol. 5, no. 5, pp. 2159-2170, 2010.

[25] G. Kasilingam, "Particle swarm optimization based pid power system stabilizer for a synchronous machine," International Journal of Electrical, Electronic Science and Engineering, vol. 8, no. 1, pp. 118-123, 2014.

[26] B. Theja, A. Rajasekhar, D. Kothari, and S. Das, "Design of PID controller based power system stabilizer using modified Philip-Heffron's model: an artificial bee colony approach," in Proceedings of the IEEE Symposium on Swarm Intelligence (SIS '13), pp. 228-234, Singapore, April 2013.

[27] K. Karthikeyan and P. Lakshmi, "Optimal design of PID controller for improving rotor angle stability using BBO," Procedia Engineering, vol. 38, pp. 889-902, 2012.

[28] P. Bera, T. Basu, and D. Das, "Design of P-I power system stabilizers for damping inter-area oscillation," in Frontiers in Computer Education, vol. 133 of Advances in Intelligent and Soft Computing, pp. 551-558, Springer, Berlin, Germany, 2012.

[29] H. Alkhatib and J. Duveau, "Dynamic genetic algorithms for robust design of multimachine power system stabilizers," International Journal of Electrical Power \& Energy Systems, vol. 45, no. 1, pp. 242-251, 2013.

[30] Y.-Y. Hsu and C.-L. Chen, "Identification of optimum location for stabiliser applications using participation factors," IEE Proceedings C: Generation Transmission and Distribution, vol. 134, no. 3, pp. 238-244, 1987.

[31] F. Demello and C. Concordia, "Concepts of synchronous machine stability as affected by excitation control," IEEE Transactions on Power Apparatus and Systems, vol. 88, no. 4, pp. 316329, 1969.

[32] M. Eslami, H. Shareef, A. Mohamed, and S. P. Ghoshal, "Tuning of power system stabilizers using particle swarm optimization with passive congregation," International Journal of Physical Sciences, vol. 5, no. 17, pp. 2574-2589, 2010.

[33] D. K. Sambariya and R. Gupta, "Fuzzy applications in a multi-machine power system stabilizer," Journal of Electrical Engineering and Technology, vol. 5, no. 3, pp. 503-510, 2010.

[34] D. K. Sambariya and R. Prasad, "Design of harmony search algorithm based tuned fuzzy logic power system stabilizer," International Review of Electrical Engineering, vol. 8, no. 5, pp. 1594-1607, 2013.

[35] D. K. Sambariya, Small signal stability enhancement using power system stabilizer [Ph.D. thesis], Department of Electrical Engineering, Indian Institute of Technology Roorkee, Roorkee, India, 2015.

[36] X. S. Yang, "A new metaheuristic bat-inspired algorithm," in Nature Inspired Cooperative Strategies for Optimization (NICSO 2010), vol. 284 of Studies in Computational Intelligence, pp. 6574, Springer, Berlin, Germany, 2010.

[37] X.-S. Yang and A. H. Gandomi, "Bat algorithm: a novel approach for global engineering optimization," Engineering Computations, vol. 29, no. 5, pp. 464-483, 2012.

[38] S. Kirkpatrick, C. D. Gelatt Jr., and M. P. Vecchi, "Optimization by simulated annealing," Science, vol. 220, no. 4598, pp. 671-680, 1983.

[39] Y. Zhang and A. Bose, "Design of wide-area damping controllers for interarea oscillations," IEEE Transactions on Power Systems, vol. 23, no. 3, pp. 1136-1143, 2008.

[40] X.-S. Yang, "Bat algorithm for multi-objective optimisation," International Journal of Bio-Inspired Computation, vol. 3, no. 5, pp. 267-274, 2011. 
[41] T. K. Das, G. K. Venayagamoorthy, and U. O. Aliyu, "Bioinspired algorithms for the design of multiple optimal power system stabilizers: SPPSO and BFA," IEEE Transactions on Industry Applications, vol. 44, no. 5, pp. 1445-1457, 2008.

[42] Y. L. Abdel-Magid and M. A. Abido, "Optimal multiobjective design of robust power system stabilizers using genetic algorithms," IEEE Transactions on Power Systems, vol. 18, no. 3, pp. 1125-1132, 2003.

[43] M. M. Linda and N. K. Nair, "Optimal design of multi-machine power system stabilizer using robust ant colony optimization technique," Transactions of the Institute of Measurement and Control, vol. 34, no. 7, pp. 829-840, 2012.

[44] H. Yassami, A. Darabi, and S. M. R. Rafiei, "Power system stabilizer design using Strength Pareto multi-objective optimization approach," Electric Power Systems Research, vol. 80, no. 7, pp. 838-846, 2010. 

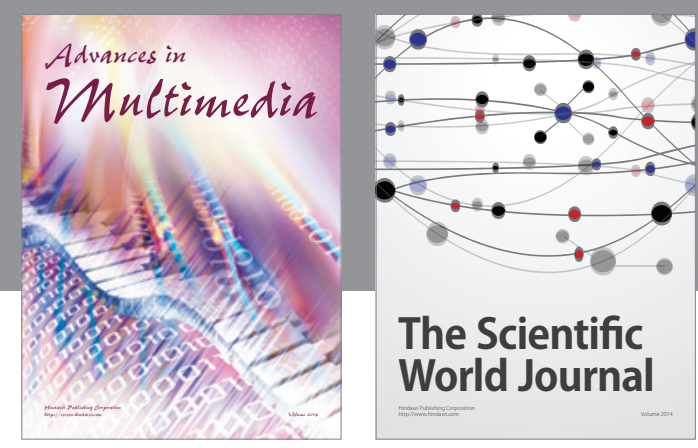

The Scientific World Journal
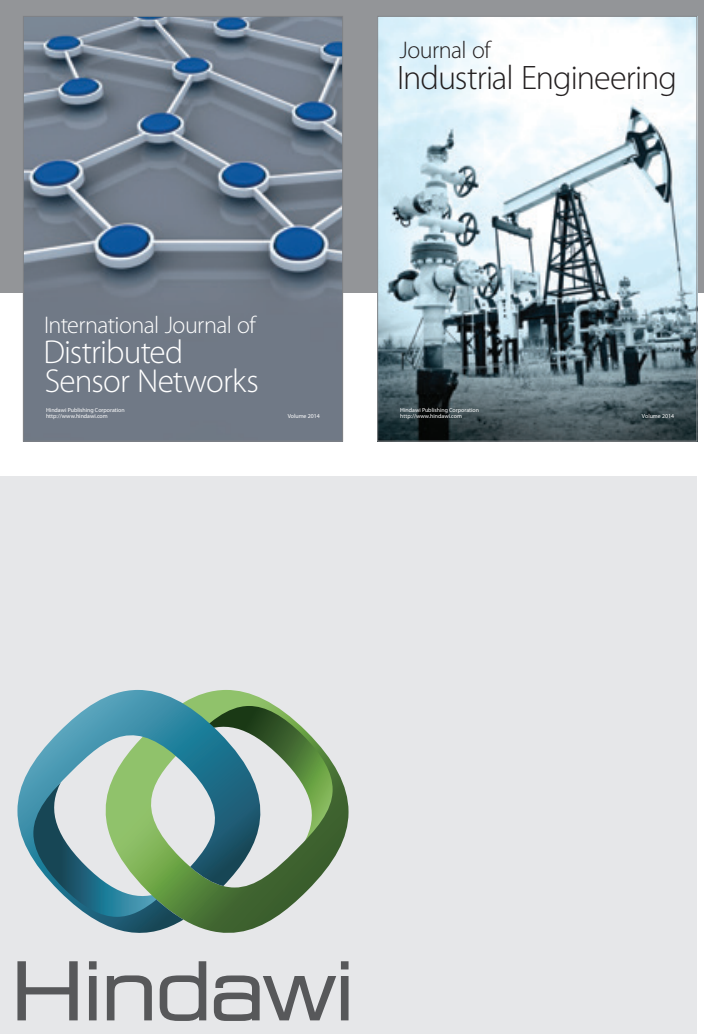

Submit your manuscripts at

http://www.hindawi.com

\section{Computer Networks} and Communications
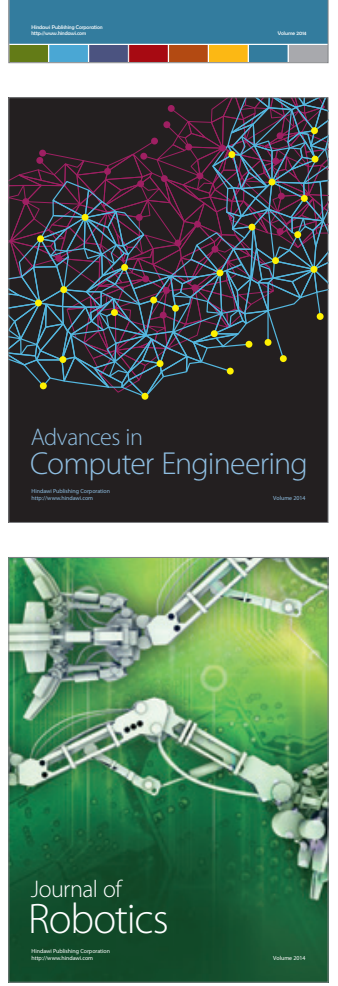
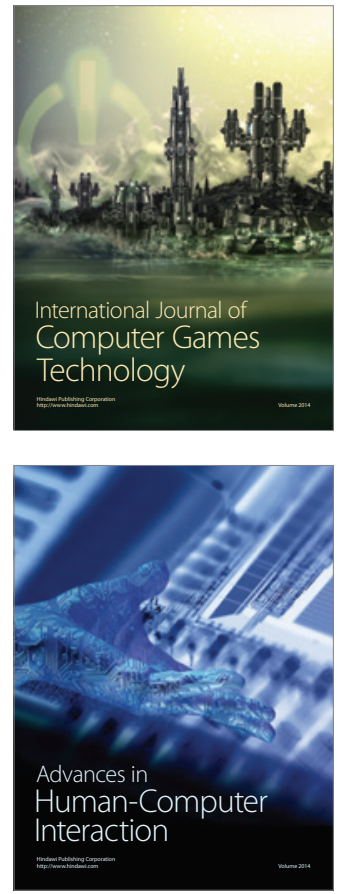
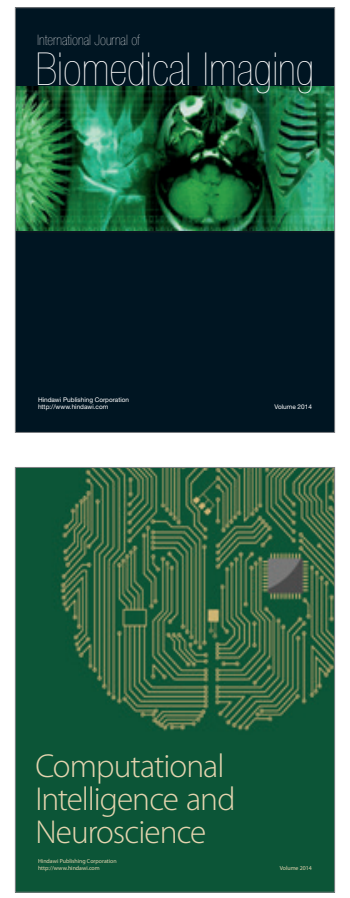
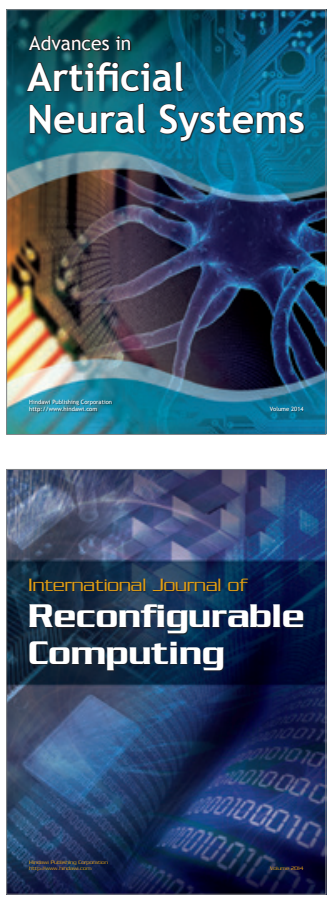
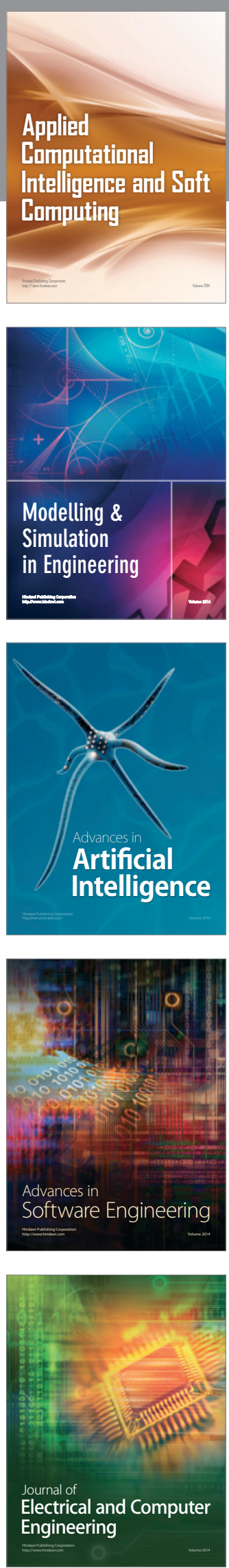